\title{
On a finite family of variational inclusions with the constraints of generalized mixed equilibrium and fixed point problems
}

\author{
Lu-Chuan Ceng ${ }^{1,2}$, Chi-Ming Chen ${ }^{3}$ and Chin-Tzong Pang ${ }^{4,5^{*}}$
}

\author{
*Correspondence: \\ imctpang@saturn.yzu.edu.tw \\ ${ }^{4}$ Department of Information \\ Management, Yuan Ze University, \\ Chung-Li, 32003, Taiwan \\ ${ }^{5}$ Innovation Center for Big Data and \\ Digital Convergence, Yuan Ze \\ University, Chung-Li, 32003, Taiwan \\ Full list of author information is \\ available at the end of the article
}

\begin{abstract}
In this paper, we introduce two iterative algorithms for finding common solutions of a finite family of variational inclusions for maximal monotone and inverse-strongly monotone mappings with the constraints of two problems: a generalized mixed equilibrium problem and a common fixed point problem of an infinite family of nonexpansive mappings and an asymptotically strict pseudocontractive mapping in the intermediate sense in a real Hilbert space. We prove some strong and weak convergence theorems for the proposed iterative algorithms under suitable conditions.

MSC: Primary 49J30; 47H09; secondary 47J20; 49M05

Keywords: generalized mixed equilibrium; variational inclusion; nonexpansive mapping; asymptotically strict pseudocontractive mapping in the intermediate sense; maximal monotone mapping; inverse-strongly monotone mapping
\end{abstract}

\section{Introduction}

Let $H$ be a real Hilbert space with inner product $\langle\cdot, \cdot\rangle$ and norm $\|\cdot\|, C$ be a nonempty closed convex subset of $H$ and $P_{C}$ be the metric projection of $H$ onto $C$. Let $S: C \rightarrow H$ be a nonlinear mapping on $C$. We denote by $\operatorname{Fix}(S)$ the set of fixed points of $S$ and by $\mathbf{R}$ the set of all real numbers. A mapping $V$ is called strongly positive on $H$ if there exists a constant $\bar{\gamma} \in(0,1]$ such that

$$
\langle V x, x\rangle \geq \bar{\gamma}\|x\|^{2}, \quad \forall x \in H .
$$

A mapping $S: C \rightarrow H$ is called $L$-Lipschitz-continuous if there exists a constant $L>0$ such that

$$
\|S x-S y\| \leq L\|x-y\|, \quad \forall x, y \in C .
$$

In particular, if $L=1$ then $S$ is called a nonexpansive mapping; if $L \in(0,1)$ then $A$ is called a contraction.

Let $\varphi: C \rightarrow \mathbf{R}$ be a real-valued function, $A: H \rightarrow H$ be a nonlinear mapping and $\Theta: C \times$ $C \rightarrow \mathbf{R}$ be a bifunction. We consider the generalized mixed equilibrium problem (GMEP)

\section{照 Springer}

@2014 Ceng et al.; licensee Springer. This is an Open Access article distributed under the terms of the Creative Commons Attribution License (http://creativecommons.org/licenses/by/2.0), which permits unrestricted use, distribution, and reproduction in any medium, provided the original work is properly cited. 
[1] of finding $x \in C$ such that

$$
\Theta(x, y)+\varphi(y)-\varphi(x)+\langle A x, y-x\rangle \geq 0, \quad \forall y \in C
$$

We denote the set of solutions of $\operatorname{GMEP}(1.1)$ by $\operatorname{GMEP}(\Theta, \varphi, A)$. The $\operatorname{GMEP}(1.1)$ is very general in the sense that it includes, as special cases, optimization problems, variational inequalities, minimax problems, Nash equilibrium problems in noncooperative games and others. The GMEP is further considered and studied in, e.g., [2-8].

Throughout this paper, it is assumed as in [1] that $\Theta: C \times C \rightarrow \mathbf{R}$ is a bifunction satisfying conditions (H1)-(H4) and $\varphi: C \rightarrow \mathbf{R}$ is a lower semicontinuous and convex function with restriction $(\mathrm{H} 5)$, where

(H1) $\Theta(x, x)=0$ for all $x \in C$

$(\mathrm{H} 2) \Theta$ is monotone, i.e., $\Theta(x, y)+\Theta(y, x) \leq 0$ for any $x, y \in C$;

(H3) $\Theta$ is upper-hemicontinuous, i.e., for each $x, y, z \in C$,

$$
\limsup _{t \rightarrow 0^{+}} \Theta(t z+(1-t) x, y) \leq \Theta(x, y)
$$

(H4) $\Theta(x, \cdot)$ is convex and lower semicontinuous for each $x \in C$;

(H5) for each $x \in H$ and $r>0$, there exist a bounded subset $D_{x} \subset C$ and $y_{x} \in C$ such that for any $z \in C \backslash D_{x}$,

$$
\Theta\left(z, y_{x}\right)+\varphi\left(y_{x}\right)-\varphi(z)+\frac{1}{r}\left\langle y_{x}-z, z-x\right\rangle<0 .
$$

Let $\Theta_{1}, \Theta_{2}: C \times C \rightarrow \mathbf{R}$ be two bifunctions, and $B_{1}, B_{2}: C \rightarrow H$ be two nonlinear mappings. Consider the system of generalized equilibrium problems (SGEP): find $\left(x^{*}, y^{*}\right) \in$ $C \times C$ such that

$$
\begin{cases}\Theta_{1}\left(x^{*}, x\right)+\left\langle B_{1} y^{*}, x-x^{*}\right\rangle+\frac{1}{\mu_{1}}\left\langle x^{*}-y^{*}, x-x^{*}\right\rangle \geq 0, & \forall x \in C, \\ \Theta_{2}\left(y^{*}, y\right)+\left\langle B_{2} x^{*}, y-y^{*}\right\rangle+\frac{1}{\mu_{2}}\left\langle y^{*}-x^{*}, y-y^{*}\right\rangle \geq 0, & \forall y \in C,\end{cases}
$$

where $\mu_{1}$ and $\mu_{2}$ are two positive constants.

Let $\left\{T_{n}\right\}_{n=1}^{\infty}$ be an infinite family of nonexpansive self-mappings on $C$ and $\left\{\lambda_{n}\right\}_{n=1}^{\infty}$ be a sequence of nonnegative numbers in [0,1]. For any $n \geq 1$, define a self-mapping $W_{n}$ on $H$ as follows:

$$
\left\{\begin{array}{l}
U_{n, n+1}=I \\
U_{n, n}=\lambda_{n} T_{n} U_{n, n+1}+\left(1-\lambda_{n}\right) I, \\
U_{n, n-1}=\lambda_{n-1} T_{n-1} U_{n, n}+\left(1-\lambda_{n-1}\right) I, \\
\vdots \\
U_{n, k}=\lambda_{k} T_{k} U_{n, k+1}+\left(1-\lambda_{k}\right) I, \\
U_{n, k-1}=\lambda_{k-1} T_{k-1} U_{n, k}+\left(1-\lambda_{k-1}\right) I \\
\vdots \\
U_{n, 2}=\lambda_{2} T_{2} U_{n, 3}+\left(1-\lambda_{2}\right) I \\
W_{n}=U_{n, 1}=\lambda_{1} T_{1} U_{n, 2}+\left(1-\lambda_{1}\right) I .
\end{array}\right.
$$

Such a mapping $W_{n}$ is called the $W$-mapping generated by $T_{n}, T_{n-1}, \ldots, T_{1}$ and $\lambda_{n}, \lambda_{n-1}$, $\ldots, \lambda_{1}$. 
Let $f: H \rightarrow H$ be a contraction and $V$ be a strongly positive bounded linear operator on $H$. Assume that $\varphi: H \rightarrow \mathbf{R}$ is a lower semicontinuous and convex functional, that $\Theta, \Theta_{1}, \Theta_{2}: H \times H \rightarrow \mathbf{R}$ satisfy conditions (H1)-(H4), and that $A, B_{1}, B_{2}: H \rightarrow H$ are inverse-strongly monotone. Very recently, motivated by Yao et al. [3], Cai and Bu [4] introduced the following hybrid extragradient-like iterative algorithm:

$$
\left\{\begin{array}{l}
z_{n}=S_{r_{n}}^{(\Theta, \varphi)}\left(x_{n}-r_{n} A x_{n}\right), \\
y_{n}=T_{\mu_{1}}^{\Theta_{1}}\left(I-\mu_{1} B_{1}\right) T_{\mu_{2}}^{\Theta_{2}}\left(I-\mu_{2} B_{2}\right) z_{n}, \\
x_{n+1}=\alpha_{n}\left(u+\gamma f\left(x_{n}\right)\right)+\beta_{n} x_{n}+\left(\left(1-\beta_{n}\right) I-\alpha_{n}(I+\mu V)\right) W_{n} y_{n}, \quad \forall n \geq 0,
\end{array}\right.
$$

for finding a common solution of GMEP (1.1), SGEP (1.2), and the fixed point problem of an infinite family of nonexpansive mappings $\left\{T_{i}\right\}_{i=1}^{\infty}$ on $H$, where $\left\{r_{n}\right\} \subset(0, \infty),\left\{\alpha_{n}\right\},\left\{\beta_{n}\right\} \subset$ $(0,1)$, and $x_{0}, u \in H$ are given. The authors proved the strong convergence of the sequence generated by the hybrid iterative algorithm (1.4) to a point $x^{*} \in\left(\bigcap_{i=1}^{\infty} \operatorname{Fix}\left(T_{i}\right)\right) \cap$ $\operatorname{GMEP}(\Theta, \varphi, A) \cap \operatorname{SGEP}(G)$ under some suitable conditions, where $\operatorname{SGEP}(G)$ is the fixed point set of the mapping $G:=T_{\mu_{1}}^{\Theta_{1}}\left(I-\mu_{1} B_{1}\right) T_{\mu_{2}}^{\Theta_{2}}\left(I-\mu_{2} B_{2}\right)$. This point $x^{*}$ also solves the following optimization problem:

$$
\min _{x \in\left(\bigcap_{n=1}^{\infty} \operatorname{Fix}\left(T_{n}\right)\right) \cap \operatorname{GMEP}(\Theta, \varphi, A) \cap \operatorname{SGEP}(G)} \frac{\mu}{2}\langle V x, x\rangle+\frac{1}{2}\|x-u\|^{2}-h(x),
$$

where $h: H \rightarrow \mathbf{R}$ is the potential function of $\gamma f$.

Let $B$ be a single-valued mapping of $C$ into $H$ and $R$ be a set-valued mapping with $D(R)=C$. Consider the following variational inclusion: find a point $x \in C$ such that

$$
0 \in B x+R x
$$

We denote by $\mathrm{I}(B, R)$ the solution set of the variational inclusion (1.5). In particular, if $B=R=0$, then $\mathrm{I}(B, R)=C$. If $B=0$, then problem (1.5) becomes the inclusion problem introduced by Rockafellar [9]. It is known that problem (1.5) provides a convenient framework for the unified study of optimal solutions in many optimization related areas including mathematical programming, complementarity problems, variational inequalities, optimal control, mathematical economics, equilibria and game theory, etc. Let a set-valued mapping $R: D(R) \subset H \rightarrow 2^{H}$ be maximal monotone. We define the resolvent operator $J_{R, \lambda}: H \rightarrow \overline{D(R)}$ associated with $R$ and $\lambda$ as follows:

$$
J_{R, \lambda}=(I+\lambda R)^{-1}, \quad \forall x \in H,
$$

where $\lambda$ is a positive number.

In 1998, Huang [10] studied problem (1.5) in the case where $R$ is maximal monotone and $B$ is strongly monotone and Lipschitz-continuous with $D(R)=C=H$. Subsequently, Zeng et al. [11] further studied problem (1.5) in the case which is more general than Huang's [10]. Moreover, the authors [11] obtained the same strong convergence conclusion as in Huang's result [10]. In addition, the authors also gave the geometric convergence rate estimate for approximate solutions. Also, various types of iterative algorithms for solving variational inclusions have been further studied and developed; for more details, refer to [5, 12-17] and the references therein. 
In 2011, for the case where $C=H$, Yao et al. [5] introduced and analyzed an iterative algorithms for finding a common element of the set of solutions of the GMEP (1.1), the set of solutions of the variational inclusion (1.5) for maximal monotone and inverse-strongly monotone mappings and the set of fixed points of a countable family of nonexpansive mappings on $H$.

Recently, Kim and $\mathrm{Xu}[18]$ introduced the concept of asymptotically $\kappa$-strict pseudocontractive mappings in a Hilbert space.

Definition 1.1 Let $C$ be a nonempty subset of a Hilbert space $H$. A mapping $S: C \rightarrow C$ is said to be an asymptotically $\kappa$-strict pseudocontractive mapping with sequence $\left\{\gamma_{n}\right\}$ if there exist a constant $\kappa \in[0,1)$ and a sequence $\left\{\gamma_{n}\right\}$ in $[0, \infty)$ with $\lim _{n \rightarrow \infty} \gamma_{n}=0$ such that

$$
\left\|S^{n} x-S^{n} y\right\|^{2} \leq\left(1+\gamma_{n}\right)\|x-y\|^{2}+\kappa\left\|x-S^{n} x-\left(y-S^{n} y\right)\right\|^{2}, \quad \forall n \geq 1, \forall x, y \in C .
$$

Subsequently, Sahu et al. [19] considered the concept of asymptotically $\kappa$-strict pseudocontractive mappings in the intermediate sense, which are not necessarily Lipschitzian.

Definition 1.2 Let $C$ be a nonempty subset of a Hilbert space $H$. A mapping $S: C \rightarrow C$ is said to be an asymptotically $\kappa$-strict pseudocontractive mapping in the intermediate sense with sequence $\left\{\gamma_{n}\right\}$ if there exist a constant $\kappa \in[0,1)$ and a sequence $\left\{\gamma_{n}\right\}$ in $[0, \infty)$ with $\lim _{n \rightarrow \infty} \gamma_{n}=0$ such that

$$
\limsup _{n \rightarrow \infty} \sup _{x, y \in C}\left(\left\|S^{n} x-S^{n} y\right\|^{2}-\left(1+\gamma_{n}\right)\|x-y\|^{2}-\kappa\left\|x-S^{n} x-\left(y-S^{n} y\right)\right\|^{2}\right) \leq 0 .
$$

Put $c_{n}:=\max \left\{0, \sup _{x, y \in C}\left(\left\|S^{n} x-S^{n} y\right\|^{2}-\left(1+\gamma_{n}\right)\|x-y\|^{2}-\kappa\left\|x-S^{n} x-\left(y-S^{n} y\right)\right\|^{2}\right)\right\}$. Then $c_{n} \geq 0(\forall n \geq 1), c_{n} \rightarrow 0(n \rightarrow \infty)$, and (1.6) reduce to the relation

$$
\left\|S^{n} x-S^{n} y\right\|^{2} \leq\left(1+\gamma_{n}\right)\|x-y\|^{2}+\kappa\left\|x-S^{n} x-\left(y-S^{n} y\right)\right\|^{2}+c_{n}, \quad \forall n \geq 1, \forall x, y \in C .
$$

Whenever $c_{n}=0$ for all $n \geq 1$ in (1.7), then $S$ is an asymptotically $\kappa$-strict pseudocontractive mapping with sequence $\left\{\gamma_{n}\right\}$. The authors [19] derived the weak and strong convergence of the modified Mann iteration processes for an asymptotically $\kappa$-strict pseudocontractive mapping in the intermediate sense with sequence $\left\{\gamma_{n}\right\}$. More precisely, they first established one weak convergence theorem for the following iterative scheme:

$$
\left\{\begin{array}{l}
x_{1}=x \in C \text { chosen arbitrarily, } \\
x_{n+1}=\left(1-\alpha_{n}\right) x_{n}+\alpha_{n} S^{n} x_{n}, \quad \forall n \geq 1,
\end{array}\right.
$$

where $0<\delta \leq \alpha_{n} \leq 1-\kappa-\delta, \sum_{n=1}^{\infty} \alpha_{n} c_{n}<\infty$, and $\sum_{n=1}^{\infty} \gamma_{n}<\infty$; and then obtained another strong convergence theorem for the following iterative scheme:

$$
\left\{\begin{array}{l}
x_{1}=x \in C \text { chosen arbitrary, } \\
y_{n}=\left(1-\alpha_{n}\right) x_{n}+\alpha_{n} S^{n} x_{n}, \\
C_{n}=\left\{z \in C:\left\|y_{n}-z\right\|^{2} \leq\left\|x_{n}-z\right\|^{2}+\theta_{n}\right\} \\
Q_{n}=\left\{z \in C:\left\langle x_{n}-z, x-x_{n}\right\rangle \geq 0\right\}, \\
x_{n+1}=P_{C_{n} \cap Q_{n}} x, \quad \forall n \geq 1,
\end{array}\right.
$$

where $0<\delta \leq \alpha_{n} \leq 1-\kappa, \theta_{n}=c_{n}+\gamma_{n} \Delta_{n}$, and $\Delta_{n}=\sup \left\{\left\|x_{n}-z\right\|^{2}: z \in \operatorname{Fix}(S)\right\}<\infty$. 
Inspired by the above facts, we in this paper introduce two iterative algorithms for finding common solutions of a finite family of variational inclusions for maximal monotone and inverse-strongly monotone mappings with the constraints of two problems: a generalized mixed equilibrium problem and a common fixed point problem of an infinite family of nonexpansive mappings and an asymptotically strict pseudocontractive mapping in the intermediate sense in a real Hilbert space. We prove some strong and weak convergence theorems for the proposed iterative algorithms under suitable conditions. The results presented in this paper are the supplement, extension, improvement, and generalization of the previously known results in this area.

\section{Preliminaries}

Throughout this paper, we assume that $H$ is a real Hilbert space whose inner product and norm are denoted by $\langle\cdot, \cdot\rangle$ and $\|\cdot\|$, respectively. Let $C$ be a nonempty closed convex subset of $H$. We write $x_{n} \rightarrow x$ to indicate that the sequence $\left\{x_{n}\right\}$ converges weakly to $x$ and $x_{n} \rightarrow x$ to indicate that the sequence $\left\{x_{n}\right\}$ converges strongly to $x$. Moreover, we use $\omega_{w}\left(x_{n}\right)$ to denote the weak $\omega$-limit set of the sequence $\left\{x_{n}\right\}$, i.e.,

$$
\omega_{w}\left(x_{n}\right):=\left\{x \in H: x_{n_{i}} \rightarrow x \text { for some subsequence }\left\{x_{n_{i}}\right\} \text { of }\left\{x_{n}\right\}\right\} .
$$

Definition 2.1 A mapping $A: C \rightarrow H$ is called

(i) monotone if

$$
\langle A x-A y, x-y\rangle \geq 0, \quad \forall x, y \in C ;
$$

(ii) $\eta$-strongly monotone if there exists a constant $\eta>0$ such that

$$
\langle A x-A y, x-y\rangle \geq \eta\|x-y\|^{2}, \quad \forall x, y \in C ;
$$

(iii) $\zeta$-inverse-strongly monotone if there exists a constant $\zeta>0$ such that

$$
\langle A x-A y, x-y\rangle \geq \zeta\|A x-A y\|^{2}, \quad \forall x, y \in C .
$$

It is easy to see that the projection $P_{C}$ is 1-inverse-strongly monotone (in short, 1-ism). Inverse-strongly monotone (also referred to as co-coercive) operators have been applied widely in solving practical problems in various fields.

Definition 2.2 A differentiable function $K: H \rightarrow \mathbf{R}$ is called:

(i) convex, if

$$
K(y)-K(x) \geq\left\langle K^{\prime}(x), y-x\right\rangle, \quad \forall x, y \in H,
$$

where $K^{\prime}(x)$ is the Frechet derivative of $K$ at $x$;

(ii) strongly convex, if there exists a constant $\sigma>0$ such that

$$
K(y)-K(x)-\left\langle K^{\prime}(x), y-x\right\rangle \geq \frac{\sigma}{2}\|x-y\|^{2}, \quad \forall x, y \in H .
$$

It is easy to see that if $K: H \rightarrow \mathbf{R}$ is a differentiable strongly convex function with constant $\sigma>0$ then $K^{\prime}: H \rightarrow H$ is strongly monotone with constant $\sigma>0$. 
The metric (or nearest point) projection from $H$ onto $C$ is the mapping $P_{C}: H \rightarrow C$ which assigns to each point $x \in H$ the unique point $P_{C} x \in C$ satisfying the property

$$
\left\|x-P_{C} x\right\|=\inf _{y \in C}\|x-y\|=: d(x, C) .
$$

Some important properties of projections are gathered in the following proposition.

Proposition 2.1 For given $x \in H$ and $z \in C$ :

(i) $z=P_{C} x \Leftrightarrow\langle x-z, y-z\rangle \leq 0, \forall y \in C$;

(ii) $z=P_{C} x \Leftrightarrow\|x-z\|^{2} \leq\|x-y\|^{2}-\|y-z\|^{2}, \forall y \in C$;

(iii) $\left\langle P_{C} x-P_{C} y, x-y\right\rangle \geq\left\|P_{C} x-P_{C} y\right\|^{2}, \forall y \in H$. (This implies that $P_{C}$ is nonexpansive and monotone.)

By using the technique of [20], we can readily obtain the following elementary result.

Proposition 2.2 (see [6, Lemma 1 and Proposition 1]) Let $C$ be a nonempty closed convex subset of a real Hilbert space $H$ and let $\varphi: C \rightarrow \mathbf{R}$ be a lower semicontinuous and convex function. Let $\Theta: C \times C \rightarrow \mathbf{R}$ be a bifunction satisfying the conditions (H1)-(H4). Assume that

(i) $K: H \rightarrow \mathbf{R}$ is strongly convex with constant $\sigma>0$ and the function $x \mapsto\left\langle y-x, K^{\prime}(x)\right\rangle$ is weakly upper semicontinuous for each $y \in H$;

(ii) for each $x \in H$ and $r>0$, there exist a bounded subset $D_{x} \subset C$ and $y_{x} \in C$ such that for any $z \in C \backslash D_{x}$,

$$
\Theta\left(z, y_{x}\right)+\varphi\left(y_{x}\right)-\varphi(z)+\frac{1}{r}\left\langle K^{\prime}(z)-K^{\prime}(x), y_{x}-z\right\rangle<0 .
$$

Then the following hold:

(a) for each $x \in H, S_{r}^{(\Theta, \varphi)}(x) \neq \emptyset$;

(b) $S_{r}^{(\Theta, \varphi)}$ is single-valued;

(c) $S_{r}^{(\Theta, \varphi)}$ is nonexpansive if $K^{\prime}$ is Lipschitz-continuous with constant $v>0$ and

$$
\left\langle K^{\prime}\left(x_{1}\right)-K^{\prime}\left(x_{2}\right), u_{1}-u_{2}\right\rangle \geq\left\langle K^{\prime}\left(u_{1}\right)-K^{\prime}\left(u_{2}\right), u_{1}-u_{2}\right\rangle, \quad \forall\left(x_{1}, x_{2}\right) \in H \times H,
$$

where $u_{i}=S_{r}^{(\Theta, \varphi)}\left(x_{i}\right)$ for $i=1,2$;

(d) for all $s, t>0$ and $x \in H$

$$
\begin{aligned}
& \left\langle K^{\prime}\left(S_{s}^{(\Theta, \varphi)} x\right)-K^{\prime}\left(S_{t}^{(\Theta, \varphi)} x\right), S_{s}^{(\Theta, \varphi)} x-S_{t}^{(\Theta, \varphi)} x\right\rangle \\
& \quad \leq \frac{s-t}{s}\left\langle K^{\prime}\left(S_{s}^{(\Theta, \varphi)} x\right)-K^{\prime}(x), S_{s}^{(\Theta, \varphi)} x-S_{t}^{(\Theta, \varphi)} x\right\rangle
\end{aligned}
$$

(e) $\operatorname{Fix}\left(S_{r}^{(\Theta, \varphi)}\right)=\operatorname{MEP}(\Theta, \varphi)$;

(f) $\operatorname{MEP}(\Theta, \varphi)$ is closed and convex.

In particular, whenever $\Theta: C \times C \rightarrow \mathbf{R}$ is a bifunction satisfying the conditions (H1)-(H4) and $K(x)=\frac{1}{2}\|x\|^{2}, \forall x \in H$, then, for any $x, y \in H$,

$$
\left\|S_{r}^{(\Theta, \varphi)} x-S_{r}^{(\Theta, \varphi)} y\right\|^{2} \leq\left\langle S_{r}^{(\Theta, \varphi)} x-S_{r}^{(\Theta, \varphi)} y, x-y\right\rangle
$$


$\left(S_{r}^{(\Theta, \varphi)}\right.$ is firmly nonexpansive) and

$$
\left\|S_{s}^{(\Theta, \varphi)} x-S_{t}^{(\Theta, \varphi)} x\right\| \leq \frac{|s-t|}{s}\left\|S_{s}^{(\Theta, \varphi)} x-x\right\|, \quad \forall s, t>0, x \in H
$$

In this case, $S_{r}^{(\Theta, \varphi)}$ is rewritten as $T_{r}^{(\Theta, \varphi)}$. If, in addition, $\varphi \equiv 0$, then $T_{r}^{(\Theta, \varphi)}$ is rewritten as $T_{r}^{\Theta}$ (see [21, Lemma 2.1] for more details).

We need some facts and tools in a real Hilbert space $H$ which are listed as lemmas below.

Lemma 2.1 Let $X$ be a real inner product space. Then we have the following inequality:

$$
\|x+y\|^{2} \leq\|x\|^{2}+2\langle y, x+y\rangle, \quad \forall x, y \in X
$$

Lemma 2.2 Let $H$ be a real Hilbert space. Then the following hold:

(a) $\|x-y\|^{2}=\|x\|^{2}-\|y\|^{2}-2\langle x-y, y\rangle$ for all $x, y \in H$;

(b) $\|\lambda x+\mu y\|^{2}=\lambda\|x\|^{2}+\mu\|y\|^{2}-\lambda \mu\|x-y\|^{2}$ for all $x, y \in H$ and $\lambda, \mu \in[0,1]$ with $\lambda+\mu=1$

(c) If $\left\{x_{n}\right\}$ is a sequence in $H$ such that $x_{n} \rightarrow x$, it follows that

$$
\limsup _{n \rightarrow \infty}\left\|x_{n}-y\right\|^{2}=\limsup _{n \rightarrow \infty}\left\|x_{n}-x\right\|^{2}+\|x-y\|^{2}, \quad \forall y \in H
$$

Lemma 2.3 ([19, Lemma 2.5]) Let $H$ be a real Hilbert space. Given a nonempty closed convex subset of $H$ and points $x, y, z \in H$ and given also a real number $a \in \mathbf{R}$, the set

$$
\left\{v \in C:\|y-v\|^{2} \leq\|x-v\|^{2}+\langle z, v\rangle+a\right\}
$$

is convex (and closed).

Lemma 2.4 ([19, Lemma 2.6]) Let $C$ be a nonempty subset of a Hilbert space $H$ and $S$ : $C \rightarrow C$ be an asymptotically $\kappa$-strict pseudocontractive mapping in the intermediate sense with sequence $\left\{\gamma_{n}\right\}$. Then

$$
\left\|S^{n} x-S^{n} y\right\| \leq \frac{1}{1-\kappa}\left(\kappa\|x-y\|+\sqrt{\left(1+(1-\kappa) \gamma_{n}\right)\|x-y\|^{2}+(1-\kappa) c_{n}}\right)
$$

for all $x, y \in C$ and $n \geq 1$.

Lemma 2.5 ([19, Lemma 2.7]) Let $C$ be a nonempty subset of a Hilbert space $H$ and $S$ : $C \rightarrow C$ be a uniformly continuous asymptotically $\kappa$-strict pseudocontractive mapping in the intermediate sense with sequence $\left\{\gamma_{n}\right\}$. Let $\left\{x_{n}\right\}$ be a sequence in $C$ such that $\| x_{n}-$ $x_{n+1} \| \rightarrow 0$ and $\left\|x_{n}-S^{n} x_{n}\right\| \rightarrow 0$ as $n \rightarrow \infty$. Then $\left\|x_{n}-S x_{n}\right\| \rightarrow 0$ as $n \rightarrow \infty$.

Lemma 2.6 (Demiclosedness principle [19, Proposition 3.1]) Let C be a nonempty closed convex subset of a Hilbert space $H$ and $S: C \rightarrow C$ be a continuous asymptotically $\kappa$-strict pseudocontractive mapping in the intermediate sense with sequence $\left\{\gamma_{n}\right\}$. Then $I-S$ is demiclosed at zero in the sense that if $\left\{x_{n}\right\}$ is a sequence in $C$ such that $x_{n} \rightarrow x \in C$ and $\limsup \sup _{m \rightarrow \infty} \lim \sup _{n \rightarrow \infty}\left\|x_{n}-S^{m} x_{n}\right\|=0$, then $(I-S) x=0$. 
Lemma 2.7 ([19, Proposition 3.2]) Let C be a nonempty closed convex subset of a Hilbert space $H$ and $S: C \rightarrow C$ be a continuous asymptotically $\kappa$-strict pseudocontractive mapping in the intermediate sense with sequence $\left\{\gamma_{n}\right\}$ such that $\operatorname{Fix}(S) \neq \emptyset$. Then $\operatorname{Fix}(S)$ is closed and convex.

Remark 2.1 Lemmas 2.6 and 2.7 give some basic properties of an asymptotically $\kappa$ strict pseudocontractive mapping in the intermediate sense with sequence $\left\{\gamma_{n}\right\}$. Moreover, Lemma 2.6 extends the demiclosedness principles studied for certain classes of nonlinear mappings; see [19] for more details.

Lemma 2.8 ([22, p.80]) Let $\left\{a_{n}\right\}_{n=1}^{\infty},\left\{b_{n}\right\}_{n=1}^{\infty}$, and $\left\{\delta_{n}\right\}_{n=1}^{\infty}$ be sequences of nonnegative real numbers satisfying the inequality

$$
a_{n+1} \leq\left(1+\delta_{n}\right) a_{n}+b_{n}, \quad \forall n \geq 1
$$

If $\sum_{n=1}^{\infty} \delta_{n}<\infty$ and $\sum_{n=1}^{\infty} b_{n}<\infty$, then $\lim _{n \rightarrow \infty} a_{n}$ exists. If, in addition, $\left\{a_{n}\right\}_{n=1}^{\infty}$ has a subsequence which converges to zero, then $\lim _{n \rightarrow \infty} a_{n}=0$.

Recall that a Banach space $X$ is said to satisfy the Opial condition [23] if, for any given sequence $\left\{x_{n}\right\} \subset X$ which converges weakly to an element $x \in X$, we have the inequality

$$
\limsup _{n \rightarrow \infty}\left\|x_{n}-x\right\|<\limsup _{n \rightarrow \infty}\left\|x_{n}-y\right\|, \quad \forall y \in X, y \neq x .
$$

It is well known in [23] that every Hilbert space $H$ satisfies the Opial condition.

Lemma 2.9 (see [24, Proposition 3.1]) Let C be a nonempty closed convex subset of a real Hilbert space $H$ and let $\left\{x_{n}\right\}$ be a sequence in $H$. Suppose that

$$
\left\|x_{n+1}-p\right\|^{2} \leq\left(1+\lambda_{n}\right)\left\|x_{n}-p\right\|^{2}+\delta_{n}, \quad \forall p \in C, n \geq 1,
$$

where $\left\{\lambda_{n}\right\}$ and $\left\{\delta_{n}\right\}$ are sequences of nonnegative real numbers such that $\sum_{n=1}^{\infty} \lambda_{n}<\infty$ and $\sum_{n=1}^{\infty} \delta_{n}<\infty$. Then $\left\{P_{C} x_{n}\right\}$ converges strongly in $C$.

Lemma 2.10 (see [25]) Let $C$ be a closed convex subset of a real Hilbert space H. Let $\left\{x_{n}\right\}$ be a sequence in $H$ and $u \in H$. Let $q=P_{C} u$. If $\left\{x_{n}\right\}$ is such that $\omega_{w}\left(x_{n}\right) \subset C$ and satisfies the condition

$$
\left\|x_{n}-u\right\| \leq\|u-q\|, \quad \text { for all } n,
$$

then $x_{n} \rightarrow q$ as $n \rightarrow \infty$.

Lemma 2.11 (see [26, Lemma 3.2]) Let $C$ be a nonempty closed convex subset of a real Hilbert space $H$. Let $\left\{T_{n}\right\}_{n=1}^{\infty}$ be a sequence of nonexpansive self-mappings on $C$ such that $\bigcap_{n=1}^{\infty} \operatorname{Fix}\left(T_{n}\right) \neq \emptyset$ and let $\left\{\lambda_{n}\right\}$ be a sequence in $(0, b]$ for some $b \in(0,1)$. Then, for every $x \in C$ and $k \geq 1$ the limit $\lim _{n \rightarrow \infty} U_{n, k} x$ exists. 
Remark 2.2 (see [27, Remark 3.1]) It can be known from Lemma 2.11 that if $D$ is a nonempty bounded subset of $C$, then for $\epsilon>0$ there exists $n_{0} \geq k$ such that for all $n>n_{0}$

$$
\sup _{x \in D}\left\|U_{n, k} x-U_{k} x\right\| \leq \epsilon
$$

Remark 2.3 (see [27, Remark 3.2]) Utilizing Lemma 2.11, we define a mapping $W: C \rightarrow C$ as follows:

$$
W x=\lim _{n \rightarrow \infty} W_{n} x=\lim _{n \rightarrow \infty} U_{n, 1} x, \quad \forall x \in C .
$$

Such a $W$ is called the $W$-mapping generated by $T_{1}, T_{2}, \ldots$ and $\lambda_{1}, \lambda_{2}, \ldots$ Since $W_{n}$ is nonexpansive, $W: C \rightarrow C$ is also nonexpansive. Indeed, observe that for each $x, y \in C$

$$
\|W x-W y\|=\lim _{n \rightarrow \infty}\left\|W_{n} x-W_{n} y\right\| \leq\|x-y\| .
$$

If $\left\{x_{n}\right\}$ is a bounded sequence in $C$, then we put $D=\left\{x_{n}: n \geq 1\right\}$. Hence, it is clear from Remark 2.2 that for an arbitrary $\epsilon>0$ there exists $N_{0} \geq 1$ such that for all $n>N_{0}$

$$
\left\|W_{n} x_{n}-W x_{n}\right\|=\left\|U_{n, 1} x_{n}-U_{1} x_{n}\right\| \leq \sup _{x \in D}\left\|U_{n, 1} x-U_{1} x\right\| \leq \epsilon
$$

This implies that

$$
\lim _{n \rightarrow \infty}\left\|W_{n} x_{n}-W x_{n}\right\|=0
$$

Lemma 2.12 (see [26, Lemma 3.3]) Let C be a nonempty closed convex subset of a real Hilbert space $H$. Let $\left\{T_{n}\right\}_{n=1}^{\infty}$ be a sequence of nonexpansive self-mappings on $C$ such that $\bigcap_{n=1}^{\infty} \operatorname{Fix}\left(T_{n}\right) \neq \emptyset$, and let $\left\{\lambda_{n}\right\}$ be a sequence in $(0, b]$ for some $b \in(0,1)$. Then $\operatorname{Fix}(W)=$ $\bigcap_{n=1}^{\infty} \operatorname{Fix}\left(T_{n}\right)$.

Lemma 2.13 (see [28, Theorem 10.4 (Demiclosedness Principle)]) Let $C$ be a nonempty closed convex subset of a real Hilbert space H. Let $T: C \rightarrow C$ be nonexpansive. Then $I-T$ is demiclosed on $C$. That is, whenever $\left\{x_{n}\right\}$ is a sequence in $C$ weakly converging to some $x \in C$ and the sequence $\left\{(I-T) x_{n}\right\}$ strongly converges to some $y$, it follows that $(I-T) x=y$. Here $I$ is the identity operator of $H$.

Recall that a set-valued mapping $R: D(R) \subset H \rightarrow 2^{H}$ is called monotone if, for all $x, y \in$ $D(R), f \in R(x)$, and $g \in R(y)$ imply

$$
\langle f-g, x-y\rangle \geq 0
$$

A set-valued mapping $R$ is called maximal monotone if $R$ is monotone and $(I+\lambda R) D(R)=$ $H$ for each $\lambda>0$, where $I$ is the identity mapping of $H$. We denote by $G(R)$ the graph of $R$. It is known that a monotone mapping $R$ is maximal if and only if, for $(x, f) \in H \times H$, $\langle f-g, x-y\rangle \geq 0$ for every $(y, g) \in G(R)$, we have $f \in R(x)$. We illustrate the concept of maximal monotone mapping with the following example. 
Let $A: C \rightarrow H$ be a monotone, $k$-Lipschitz-continuous mapping and let $N_{C} v$ be the normal cone to $C$ at $v \in C$, i.e.,

$$
N_{C} v=\{w \in H:\langle v-u, w\rangle \geq 0, \forall u \in C\} .
$$

Define

$$
T v= \begin{cases}A v+N_{C} v & \text { if } v \in C, \\ \varnothing & \text { if } v \notin C .\end{cases}
$$

Then $T$ is maximal monotone and $0 \in T v$ if and only if $\langle A v, y-v\rangle \geq 0$ for all $y \in C$ (see [9]).

Assume that $R: D(R) \subset H \rightarrow 2^{H}$ is a maximal monotone mapping. Let $\lambda>0$. In terms of Huang [10] (see also [11]), we have the following property for the resolvent operator $J_{R, \lambda}: H \rightarrow \overline{D(R)}$.

Lemma 2.14 $J_{R, \lambda}$ is single-valued and firmly nonexpansive, i.e.,

$$
\left\langle J_{R, \lambda} x-J_{R, \lambda} y, x-y\right\rangle \geq\left\|J_{R, \lambda} x-J_{R, \lambda} y\right\|^{2}, \quad \forall x, y \in H .
$$

Consequently, $J_{R, \lambda}$ is nonexpansive and monotone.

Lemma 2.15 (see [14]) Let $R$ be a maximal monotone mapping with $D(R)=C$. Then for any given $\lambda>0, u \in C$ is a solution of problem (1.6) if and only if $u \in C$ satisfies

$$
u=J_{R, \lambda}(u-\lambda B u) .
$$

Lemma 2.16 (see [11]) Let $R$ be a maximal monotone mapping with $D(R)=C$ and let $B: C \rightarrow H$ be a strongly monotone, continuous, and single-valued mapping. Then for each $z \in H$, the equation $z \in(B+\lambda R) x$ has a unique solution $x_{\lambda}$ for $\lambda>0$.

Lemma 2.17 (see [14]) Let $R$ be a maximal monotone mapping with $D(R)=C$ and $B: C \rightarrow$ $H$ be a monotone, continuous and single-valued mapping. Then $(I+\lambda(R+B)) C=H$ for each $\lambda>0$. In this case, $R+B$ is maximal monotone.

Lemma 2.18 (see [29]) Let $C$ be a nonempty closed convex subset of a real Hilbert space $H$, and $g: C \rightarrow \mathbf{R} \cup+\infty$ be a proper lower semicontinuous differentiable convex function. If $x^{*}$ is a solution the minimization problem

$$
g\left(x^{*}\right)=\inf _{x \in C} g(x)
$$

then

$$
\left\langle g^{\prime}(x), x-x^{*}\right\rangle \geq 0, \quad \forall x \in C .
$$

In particular, if $x^{*}$ solves (OP1), then

$$
\left\langle u+(\gamma f-(I+\mu V)) x^{*}, x-x^{*}\right\rangle \leq 0 .
$$




\section{Strong convergence theorems}

In this section, we introduce and analyze an iterative algorithm for finding common solutions of a finite family of variational inclusions for maximal monotone and inversestrongly monotone mappings with the constraints of two problems: a generalized mixed equilibrium problem and a common fixed point problem of an infinite family of nonexpansive mappings and an asymptotically strict pseudocontractive mapping in the intermediate sense in a real Hilbert space. Under appropriate conditions imposed on the parameter sequences we will prove strong convergence of the proposed algorithm.

Theorem 3.1 Let $C$ be a nonempty closed convex subset of a real Hilbert space H. Let $N$ be an integer. Let $\Theta$ be a bifunction from $C \times C$ to $\mathbf{R}$ satisfying $(\mathrm{H} 1)-(\mathrm{H} 4)$ and $\varphi: C \rightarrow \mathbf{R}$ be a lower semicontinuous and convex functional. Let $R_{i}: C \rightarrow 2^{H}$ be a maximal monotone mapping and let $A: H \rightarrow H$ and $B_{i}: C \rightarrow H$ be $\zeta$-inverse-strongly monotone and $\eta_{i}$ inverse-strongly monotone, respectively, where $i \in\{1,2, \ldots, N\}$. Let $S: C \rightarrow C$ be a uniformly continuous asymptotically $\kappa$-strict pseudocontractive mapping in the intermediate sense for some $0 \leq \kappa<1$ with sequence $\left\{\gamma_{n}\right\} \subset[0, \infty)$ such that $\lim _{n \rightarrow \infty} \gamma_{n}=0$ and $\left\{c_{n}\right\} \subset[0, \infty)$ such that $\lim _{n \rightarrow \infty} c_{n}=0$. Let $\left\{T_{n}\right\}_{n=1}^{\infty}$ be a sequence of nonexpansive self-mappings on $C$ and $\left\{\lambda_{n}\right\}$ be a sequence in $(0, b]$ for some $b \in(0,1)$. Let $V$ be a $\bar{\gamma}$-strongly positive bounded linear operator and $f: H \rightarrow H$ be an l-Lipschitzian mapping with $\gamma l<(1+\mu) \bar{\gamma}$. Assume that $\Omega:=\left(\bigcap_{n=1}^{\infty} \operatorname{Fix}\left(T_{n}\right)\right) \cap \operatorname{GMEP}(\Theta, \varphi, A) \cap\left(\bigcap_{i=1}^{N} \mathrm{I}\left(B_{i}, R_{i}\right)\right) \cap \operatorname{Fix}(S)$ is nonempty and bounded. Let $W_{n}$ be the $W$-mapping defined by (1.4) and $\left\{\alpha_{n}\right\},\left\{\beta_{n}\right\}$ and $\left\{\delta_{n}\right\}$ be three sequences in $(0,1)$ such that $\lim _{n \rightarrow \infty} \alpha_{n}=0$ and $\kappa \leq \delta_{n} \leq d<1$. Assume that:

(i) $K: H \rightarrow \mathbf{R}$ is strongly convex with constant $\sigma>0$ and its derivative $K^{\prime}$ is Lipschitz-continuous with constant $v>0$ such that the function $x \mapsto\left\langle y-x, K^{\prime}(x)\right\rangle$ is weakly upper semicontinuous for each $y \in H$;

(ii) for each $x \in H$, there exist a bounded subset $D_{x} \subset C$ and $z_{x} \in C$ such that for any $y \notin D_{x}$,

$$
\Theta\left(y, z_{x}\right)+\varphi\left(z_{x}\right)-\varphi(y)+\frac{1}{r}\left\langle K^{\prime}(y)-K^{\prime}(x), z_{x}-y\right\rangle<0 ;
$$

(iii) $0<\liminf _{n \rightarrow \infty} \beta_{n} \leq \limsup _{n \rightarrow \infty} \beta_{n}<1$;

(iv) $\left\{\lambda_{i, n}\right\} \subset\left[a_{i}, b_{i}\right] \subset\left(0,2 \eta_{i}\right), \forall i \in\{1,2, \ldots, N\}$, and $\left\{r_{n}\right\} \subset[0,2 \zeta]$ satisfies

$$
0<\liminf _{n \rightarrow \infty} r_{n} \leq \limsup _{n \rightarrow \infty} r_{n}<2 \zeta
$$

Pick any $x_{0} \in H$ and set $C_{1}=C, x_{1}=P_{C_{1}} x_{0}$. Let $\left\{x_{n}\right\}$ be a sequence generated by the following algorithm:

$$
\left\{\begin{array}{l}
u_{n}=S_{r_{n}}^{(\Theta, \varphi)}\left(I-r_{n} A\right) x_{n}, \\
z_{n}=J_{R_{N}, \lambda_{N, n}}\left(I-\lambda_{N, n} B_{N}\right) J_{R_{N-1}, \lambda_{N-1, n}}\left(I-\lambda_{N-1, n} B_{N-1}\right) \cdots J_{R_{1}, \lambda_{1, n}}\left(I-\lambda_{1, n} B_{1}\right) u_{n}, \\
k_{n}=\delta_{n} z_{n}+\left(1-\delta_{n}\right) S^{n} z_{n}, \\
y_{n}=\alpha_{n}\left(u+\gamma f\left(x_{n}\right)\right)+\beta_{n} k_{n}+\left(\left(1-\beta_{n}\right) I-\alpha_{n}(I+\mu V)\right) W_{n} z_{n}, \\
C_{n+1}=\left\{z \in C_{n}:\left\|y_{n}-z\right\|^{2} \leq\left\|x_{n}-z\right\|^{2}+\theta_{n}\right\}, \\
x_{n+1}=P_{C_{n+1}} x_{0}, \quad \forall n \geq 0,
\end{array}\right.
$$

where $\theta_{n}=\left(\alpha_{n}+\gamma_{n}\right) \Delta_{n} \varrho+c_{n} \varrho, \Delta_{n}=\sup \left\{\left\|x_{n}-p\right\|^{2}+\|u+(\gamma f-I-\mu V) p\|^{2}: p \in \Omega\right\}<\infty$, and $\varrho=\frac{1}{1-\sup _{n \geq 1} \alpha_{n}}<\infty$. If $S_{r}^{(\Theta, \varphi)}$ is firmly nonexpansive, then the following statements hold: 
(I) $\left\{x_{n}\right\}$ converges strongly to $P_{\Omega} x_{0}$;

(II) $\left\{x_{n}\right\}$ converges strongly to $P_{\Omega} x_{0}$, which solves the optimization problem

$$
\min _{x \in \Omega} \frac{\mu}{2}\langle V x, x\rangle+\frac{1}{2}\|x-u\|^{2}-h(x)
$$

provided $\gamma_{n}+c_{n}+\left\|x_{n}-y_{n}\right\|=o\left(\alpha_{n}\right)$ additionally, where $h: H \rightarrow \mathbf{R}$ is the potential function of $\gamma f$.

Proof Since $\lim _{n \rightarrow \infty} \alpha_{n}=0$ and $0<\liminf _{n \rightarrow \infty} \beta_{n} \leq \lim \sup _{n \rightarrow \infty} \beta_{n}<1$, we may assume, without loss of generality, that $\alpha_{n} \leq\left(1-\beta_{n}\right)(1+\mu\|V\|)^{-1}$. Since $V$ is a $\bar{\gamma}$-strongly positive bounded linear operator on $H$, we know that

$$
\|V\|=\sup \{\langle V u, u\rangle: u \in H,\|u\|=1\} .
$$

Observe that

$$
\begin{aligned}
\left\langle\left(\left(1-\beta_{n}\right) I-\alpha_{n}(I+\mu V)\right) u, u\right\rangle & =1-\beta_{n}-\alpha_{n}-\alpha_{n} \mu\langle V u, u\rangle \\
& \geq 1-\beta_{n}-\alpha_{n}-\alpha_{n} \mu\|V\|
\end{aligned}
$$$$
\geq 0 \text {, }
$$

that is, $\left(1-\beta_{n}\right) I-\alpha_{n}(I+\mu V)$ is positive. It follows that

$$
\begin{aligned}
\left\|\left(1-\beta_{n}\right) I-\alpha_{n}(I+\mu V)\right\| & =\sup \left\{\left\langle\left(\left(1-\beta_{n}\right) I-\alpha_{n}(I+\mu V)\right) u, u\right\rangle: u \in H,\|u\|=1\right\} \\
& =\sup \left\{1-\beta_{n}-\alpha_{n}-\alpha_{n} \mu\langle V u, u\rangle: u \in H,\|u\|=1\right\} \\
& \leq 1-\beta_{n}-\alpha_{n}-\alpha_{n} \mu \bar{\gamma} .
\end{aligned}
$$

Put

$$
\Lambda_{n}^{i}=J_{R_{i}, \lambda_{i, n}}\left(I-\lambda_{i, n} B_{i}\right) J_{R_{i-1}, \lambda_{i-1, n}}\left(I-\lambda_{i-1, n} B_{i-1}\right) \cdots J_{R_{1}, \lambda_{1, n}}\left(I-\lambda_{1, n} B_{1}\right)
$$

for all $i \in\{1,2, \ldots, N\}$ and $n \geq 1$, and $\Lambda_{n}^{0}=I$, where $I$ is the identity mapping on $H$. Then we have that $z_{n}=\Lambda_{n}^{N} u_{n}$. We divide the rest of the proof into several steps.

Step 1. We show that $\left\{x_{n}\right\}$ is well defined. It is obvious that $C_{n}$ is closed and convex. As the defining inequality in $C_{n}$ is equivalent to the inequality

$$
\left\langle 2\left(x_{n}-z_{n}\right), z\right\rangle \leq\left\|x_{n}\right\|^{2}-\left\|z_{n}\right\|^{2}+\theta_{n},
$$

by Lemma 2.3 we know that $C_{n}$ is convex and closed for every $n \geq 1$.

First of all, we show that $\Omega \subset C_{n}$ for all $n \geq 1$. Suppose that $\Omega \subset C_{n}$ for some $n \geq 1$. Take $p \in \Omega$ arbitrarily. Since $p=S_{r_{n}}^{(\Theta, \varphi)}\left(p-r_{n} A p\right), A$ is $\zeta$-inverse strongly monotone and $0 \leq r_{n} \leq 2 \zeta$, we have

$$
\begin{aligned}
\left\|u_{n}-p\right\|^{2} & =\left\|S_{r_{n}}^{(\Theta, \varphi)}\left(I-r_{n} A\right) x_{n}-S_{r_{n}}^{(\Theta, \varphi)}\left(I-r_{n} A\right) p\right\|^{2} \\
& \leq\left\|\left(I-r_{n} A\right) x_{n}-\left(I-r_{n} A\right) p\right\|^{2}
\end{aligned}
$$




$$
\begin{aligned}
& =\left\|\left(x_{n}-p\right)-r_{n}\left(A x_{n}-A p\right)\right\|^{2} \\
& =\left\|x_{n}-p\right\|^{2}-2 r_{n}\left\langle x_{n}-p, A x_{n}-A p\right\rangle+r_{n}^{2}\left\|A x_{n}-A p\right\|^{2} \\
& \leq\left\|x_{n}-p\right\|^{2}-2 r_{n} \zeta\left\|A x_{n}-A p\right\|^{2}+r_{n}^{2}\left\|A x_{n}-A p\right\|^{2} \\
& =\left\|x_{n}-p\right\|^{2}+r_{n}\left(r_{n}-2 \zeta\right)\left\|A x_{n}-A p\right\|^{2} \\
& \leq\left\|x_{n}-p\right\|^{2} .
\end{aligned}
$$

Since $p=J_{R_{i}, \lambda_{i, n}}\left(I-\lambda_{i, n} B_{i}\right) p, \Lambda_{n}^{i} p=p$, and $B_{i}$ is $\eta_{i}$-inverse-strongly monotone, where $\eta_{i} \in$ $\left(0,2 \eta_{i}\right), i \in\{1,2, \ldots, N\}$, by Lemma 2.14 we deduce that

$$
\begin{aligned}
\left\|z_{n}-p\right\|^{2} & =\left\|J_{R_{N}, \lambda_{N, n}}\left(I-\lambda_{N, n} B_{N}\right) \Lambda_{n}^{N-1} u_{n}-J_{R_{N}, \lambda_{N, n}}\left(I-\lambda_{N, n} B_{N}\right) \Lambda_{n}^{N-1} p\right\|^{2} \\
& \leq\left\|\left(I-\lambda_{N, n} B_{N}\right) \Lambda_{n}^{N-1} u_{n}-\left(I-\lambda_{N, n} B_{N}\right) \Lambda_{n}^{N-1} p\right\|^{2} \\
& =\left\|\left(\Lambda_{n}^{N-1} u_{n}-\Lambda_{n}^{N-1} p\right)-\lambda_{N, n}\left(B_{N} \Lambda_{n}^{N-1} u_{n}-B_{N} \Lambda_{n}^{N-1} p\right)\right\|^{2} \\
& \leq\left\|\Lambda_{n}^{N-1} u_{n}-\Lambda_{n}^{N-1} p\right\|^{2}+\lambda_{N, n}\left(\lambda_{N, n}-2 \eta_{N}\right)\left\|B_{N} \Lambda_{n}^{N-1} u_{n}-B_{N} \Lambda_{n}^{N-1} p\right\|^{2} \\
& \leq\left\|\Lambda_{n}^{N-1} u_{n}-\Lambda_{n}^{N-1} p\right\|^{2} \\
& \vdots \\
& \leq\left\|\Lambda_{n}^{0} u_{n}-\Lambda_{n}^{0} p\right\|^{2} \\
& =\left\|u_{n}-p\right\|^{2} .
\end{aligned}
$$

Combining (3.2) and (3.3), we have

$$
\left\|z_{n}-p\right\| \leq\left\|x_{n}-p\right\|
$$

By Lemma 2.2(b), we deduce from (3.1) and (3.4) that

$$
\begin{aligned}
\left\|k_{n}-p\right\|^{2}= & \left\|\delta_{n}\left(z_{n}-p\right)+\left(1-\delta_{n}\right)\left(S^{n} z_{n}-p\right)\right\|^{2} \\
= & \delta_{n}\left\|z_{n}-p\right\|^{2}+\left(1-\delta_{n}\right)\left\|S^{n} z_{n}-p\right\|^{2}-\delta_{n}\left(1-\delta_{n}\right)\left\|z_{n}-S^{n} z_{n}\right\|^{2} \\
\leq & \delta_{n}\left\|z_{n}-p\right\|^{2}+\left(1-\delta_{n}\right)\left[\left(1+\gamma_{n}\right)\left\|z_{n}-p\right\|^{2}\right. \\
& \left.+\kappa\left\|z_{n}-S^{n} z_{n}\right\|^{2}+c_{n}\right]-\delta_{n}\left(1-\delta_{n}\right)\left\|z_{n}-S^{n} z_{n}\right\|^{2} \\
= & {\left[1+\gamma_{n}\left(1-\delta_{n}\right)\right]\left\|z_{n}-p\right\|^{2}+\left(1-\delta_{n}\right)\left(\kappa-\delta_{n}\right)\left\|z_{n}-S^{n} z_{n}\right\|^{2}+\left(1-\delta_{n}\right) c_{n} } \\
\leq & \left(1+\gamma_{n}\right)\left\|z_{n}-p\right\|^{2}+\left(1-\delta_{n}\right)\left(\kappa-\delta_{n}\right)\left\|z_{n}-S^{n} z_{n}\right\|^{2}+c_{n} \\
\leq & \left(1+\gamma_{n}\right)\left\|z_{n}-p\right\|^{2}+c_{n} .
\end{aligned}
$$

Set $\bar{V}=I+\mu V$. Then, for $\gamma l \leq(1+\mu) \bar{\gamma}$, by Lemma 2.1 we obtain from (3.1), (3.4), and (3.5)

$$
\begin{aligned}
& \left\|y_{n}-p\right\|^{2} \\
& \quad=\left\|\alpha_{n}\left(u+\gamma f\left(x_{n}\right)\right)+\beta_{n} k_{n}+\left(\left(1-\beta_{n}\right) I-\alpha_{n} \bar{V}\right) W_{n} z_{n}-p\right\|^{2}
\end{aligned}
$$




$$
\begin{aligned}
& =\left\|\alpha_{n}\left(u+\gamma f\left(x_{n}\right)-\bar{V} p\right)+\beta_{n}\left(k_{n}-p\right)+\left(\left(1-\beta_{n}\right) I-\alpha_{n} \bar{V}\right)\left(W_{n} z_{n}-p\right)\right\|^{2} \\
& =\| \alpha_{n}(u+\gamma f(p)-\bar{V} p)+\alpha_{n} \gamma\left(f\left(x_{n}\right)-f(p)\right) \\
& +\beta_{n}\left(k_{n}-p\right)+\left(\left(1-\beta_{n}\right) I-\alpha_{n} \bar{V}\right)\left(W_{n} z_{n}-p\right) \|^{2} \\
& \leq\left\|\alpha_{n} \gamma\left(f\left(x_{n}\right)-f(p)\right)+\beta_{n}\left(k_{n}-p\right)+\left(\left(1-\beta_{n}\right) I-\alpha_{n} \bar{V}\right)\left(W_{n} z_{n}-p\right)\right\|^{2} \\
& +2 \alpha_{n}\left\langle(u+\gamma f(p)-\bar{V} p), y_{n}-p\right\rangle \\
& \leq\left[\alpha_{n} \gamma\left\|f\left(x_{n}\right)-f(p)\right\|+\beta_{n}\left\|k_{n}-p\right\|+\left\|\left(\left(1-\beta_{n}\right) I-\alpha_{n} \bar{V}\right)\left(W_{n} z_{n}-p\right)\right\|\right]^{2} \\
& +2 \alpha_{n}\left\langle(u+\gamma f(p)-\bar{V} p), y_{n}-p\right\rangle \\
& \leq\left[\alpha_{n} \gamma l\left\|x_{n}-p\right\|+\beta_{n}\left\|k_{n}-p\right\|+\left(1-\beta_{n}-\alpha_{n}-\alpha_{n} \mu \bar{\gamma}\right)\left\|W_{n} z_{n}-p\right\|\right]^{2} \\
& +2 \alpha_{n}\|u+\gamma f(p)-\bar{V} p\|\left\|y_{n}-p\right\| \\
& \leq\left[\alpha_{n}(1+\mu) \bar{\gamma}\left\|x_{n}-p\right\|+\beta_{n}\left\|k_{n}-p\right\|+\left(1-\beta_{n}-\alpha_{n}(1+\mu) \bar{\gamma}\right)\left\|z_{n}-p\right\|\right]^{2} \\
& +\alpha_{n}\left(\|u+\gamma f(p)-\bar{V} p\|^{2}+\left\|y_{n}-p\right\|^{2}\right) \\
& \leq \alpha_{n}(1+\mu) \bar{\gamma}\left\|x_{n}-p\right\|^{2}+\beta_{n}\left\|k_{n}-p\right\|^{2}+\left(1-\beta_{n}-\alpha_{n}(1+\mu) \bar{\gamma}\right)\left\|z_{n}-p\right\|^{2} \\
& +\alpha_{n}\left(\|u+\gamma f(p)-\bar{V} p\|^{2}+\left\|y_{n}-p\right\|^{2}\right) \\
& \leq \alpha_{n}(1+\mu) \bar{\gamma}\left\|x_{n}-p\right\|^{2}+\beta_{n}\left(\left(1+\gamma_{n}\right)\left\|z_{n}-p\right\|^{2}+c_{n}\right) \\
& +\left(1-\beta_{n}-\alpha_{n}(1+\mu) \bar{\gamma}\right)\left\|z_{n}-p\right\|^{2}+\alpha_{n}\left(\|u+\gamma f(p)-\bar{V} p\|^{2}+\left\|y_{n}-p\right\|^{2}\right) \\
& \leq \alpha_{n}(1+\mu) \bar{\gamma}\left\|x_{n}-p\right\|^{2}+\beta_{n}\left(\left(1+\gamma_{n}\right)\left\|z_{n}-p\right\|^{2}+c_{n}\right) \\
& +\left(1-\beta_{n}-\alpha_{n}(1+\mu) \bar{\gamma}\right)\left(\left(1+\gamma_{n}\right)\left\|z_{n}-p\right\|^{2}+c_{n}\right) \\
& +\alpha_{n}\left(\|u+\gamma f(p)-\bar{V} p\|^{2}+\left\|y_{n}-p\right\|^{2}\right) \\
& =\alpha_{n}(1+\mu) \bar{\gamma}\left\|x_{n}-p\right\|^{2}+\left(1-\alpha_{n}(1+\mu) \bar{\gamma}\right)\left(\left(1+\gamma_{n}\right)\left\|z_{n}-p\right\|^{2}+c_{n}\right) \\
& +\alpha_{n}\left(\|u+\gamma f(p)-\bar{V} p\|^{2}+\left\|y_{n}-p\right\|^{2}\right) \\
& \leq \alpha_{n}(1+\mu) \bar{\gamma}\left(\left(1+\gamma_{n}\right)\left\|x_{n}-p\right\|^{2}+c_{n}\right) \\
& +\left(1-\alpha_{n}(1+\mu) \bar{\gamma}\right)\left(\left(1+\gamma_{n}\right)\left\|x_{n}-p\right\|^{2}+c_{n}\right) \\
& +\alpha_{n}\left(\|u+\gamma f(p)-\bar{V} p\|^{2}+\left\|y_{n}-p\right\|^{2}\right) \\
& =\left(1+\gamma_{n}\right)\left\|x_{n}-p\right\|^{2}+c_{n}+\alpha_{n}\left(\|u+\gamma f(p)-\bar{V} p\|^{2}+\left\|y_{n}-p\right\|^{2}\right),
\end{aligned}
$$

which hence yields

$$
\begin{aligned}
\left\|y_{n}-p\right\|^{2} & \leq \frac{1+\gamma_{n}}{1-\alpha_{n}}\left\|x_{n}-p\right\|^{2}+\frac{\alpha_{n}}{1-\alpha_{n}}\|u+\gamma f(p)-\bar{V} p\|^{2}+\frac{1}{1-\alpha_{n}} c_{n} \\
& =\left(1+\frac{\alpha_{n}+\gamma_{n}}{1-\alpha_{n}}\right)\left\|x_{n}-p\right\|^{2}+\frac{\alpha_{n}}{1-\alpha_{n}}\|u+\gamma f(p)-\bar{V} p\|^{2}+\frac{1}{1-\alpha_{n}} c_{n} \\
& \leq\left(1+\frac{\alpha_{n}+\gamma_{n}}{1-\alpha_{n}}\right)\left\|x_{n}-p\right\|^{2}+\frac{\alpha_{n}+\gamma_{n}}{1-\alpha_{n}}\|u+\gamma f(p)-\bar{V} p\|^{2}+\frac{1}{1-\alpha_{n}} c_{n} \\
& =\left\|x_{n}-p\right\|^{2}+\frac{\alpha_{n}+\gamma_{n}}{1-\alpha_{n}}\left(\left\|x_{n}-p\right\|^{2}+\|u+\gamma f(p)-\bar{V} p\|^{2}\right)+\frac{1}{1-\alpha_{n}} c_{n}
\end{aligned}
$$




$$
\begin{aligned}
& \leq\left\|x_{n}-p\right\|^{2}+\left(\alpha_{n}+\gamma_{n}\right) \varrho\left(\left\|x_{n}-p\right\|^{2}+\|u+\gamma f(p)-\bar{V} p\|^{2}\right)+\varrho c_{n} \\
& \leq\left\|x_{n}-p\right\|^{2}+\left(\alpha_{n}+\gamma_{n}\right) \Delta_{n} \varrho+c_{n} \varrho \\
& =\left\|x_{n}-p\right\|^{2}+\theta_{n},
\end{aligned}
$$

where $\theta_{n}=\left(\alpha_{n}+\gamma_{n}\right) \Delta_{n} \varrho+c_{n} \varrho, \Delta_{n}=\sup \left\{\left\|x_{n}-p\right\|^{2}+\|u+\gamma f(p)-\bar{V} p\|^{2}: p \in \Omega\right\}<\infty$, and $\varrho=\frac{1}{1-\sup _{n \geq 1} \alpha_{n}}<\infty$ (due to $\left\{\alpha_{n}\right\} \subset(0,1)$ and $\lim _{n \rightarrow \infty} \alpha_{n}=0$ ). Hence $p \in C_{n+1}$. This implies that $\Omega \subset C_{n}$ for all $n \geq 1$. Therefore, $\left\{x_{n}\right\}$ is well defined.

Step 2. We prove that $\left\|x_{n}-k_{n}\right\| \rightarrow 0$ as $n \rightarrow \infty$.

Indeed, let $v=P_{\Omega} x_{0}$. From $x_{n}=P_{C_{n}} x_{0}$ and $v \in \Omega \subset C_{n}$, we obtain

$$
\left\|x_{n}-x_{0}\right\| \leq\left\|v-x_{0}\right\|
$$

This implies that $\left\{x_{n}\right\}$ is bounded and hence $\left\{u_{n}\right\},\left\{z_{n}\right\},\left\{k_{n}\right\}$, and $\left\{y_{n}\right\}$ are also bounded. Since $x_{n+1} \in C_{n+1} \subset C_{n}$ and $x_{n}=P_{C_{n}} x_{0}$, we have

$$
\left\|x_{n}-x_{0}\right\| \leq\left\|x_{n+1}-x_{0}\right\|, \quad \forall n \geq 1
$$

Therefore $\lim _{n \rightarrow \infty}\left\|x_{n}-x_{0}\right\|$ exists. From $x_{n}=P_{C_{n}} x_{0}, x_{n+1} \in C_{n+1} \subset C_{n}$, by Proposition 2.1(ii) we obtain

$$
\left\|x_{n+1}-x_{n}\right\|^{2} \leq\left\|x_{0}-x_{n+1}\right\|^{2}-\left\|x_{0}-x_{n}\right\|^{2},
$$

which implies

$$
\lim _{n \rightarrow \infty}\left\|x_{n+1}-x_{n}\right\|=0
$$

It follows from $x_{n+1} \in C_{n+1}$ that $\left\|y_{n}-x_{n+1}\right\|^{2} \leq\left\|x_{n}-x_{n+1}\right\|^{2}+\theta_{n}$ and hence

$$
\begin{aligned}
\left\|x_{n}-y_{n}\right\|^{2} & \leq 2\left(\left\|x_{n}-x_{n+1}\right\|^{2}+\left\|x_{n+1}-y_{n}\right\|^{2}\right) \\
& \leq 2\left(\left\|x_{n}-x_{n+1}\right\|^{2}+\left\|x_{n}-x_{n+1}\right\|^{2}+\theta_{n}\right) \\
& =2\left(2\left\|x_{n}-x_{n+1}\right\|^{2}+\theta_{n}\right) .
\end{aligned}
$$

From (3.8) and $\lim _{n \rightarrow \infty} \theta_{n}=0$, we have

$$
\lim _{n \rightarrow \infty}\left\|x_{n}-y_{n}\right\|=0
$$

Also, utilizing Lemmas 2.1 and 2.2(b) we obtain from (3.1), (3.4), and (3.5)

$$
\begin{aligned}
\| y_{n}- & p \|^{2} \\
= & \left\|\alpha_{n}\left(u+\gamma f\left(x_{n}\right)-\bar{V} W_{n} z_{n}\right)+\beta_{n}\left(k_{n}-p\right)+\left(1-\beta_{n}\right)\left(W_{n} z_{n}-p\right)\right\|^{2} \\
\leq & \left\|\beta_{n}\left(k_{n}-p\right)+\left(1-\beta_{n}\right)\left(W_{n} z_{n}-p\right)\right\|^{2}+2 \alpha_{n}\left\langle u+\gamma f\left(x_{n}\right)-\bar{V} W_{n} z_{n}, y_{n}-p\right\rangle \\
= & \beta_{n}\left\|k_{n}-p\right\|^{2}+\left(1-\beta_{n}\right)\left\|W_{n} z_{n}-p\right\|^{2}-\beta_{n}\left(1-\beta_{n}\right)\left\|k_{n}-W_{n} z_{n}\right\|^{2} \\
& +2 \alpha_{n}\left\|u+\gamma f\left(x_{n}\right)-\bar{V} W_{n} z_{n}\right\|\left\|y_{n}-p\right\|
\end{aligned}
$$




$$
\begin{aligned}
\leq & \beta_{n}\left\|k_{n}-p\right\|^{2}+\left(1-\beta_{n}\right)\left\|z_{n}-p\right\|^{2}-\beta_{n}\left(1-\beta_{n}\right)\left\|k_{n}-W_{n} z_{n}\right\|^{2} \\
& +2 \alpha_{n}\left\|u+\gamma f\left(x_{n}\right)-\bar{V} W_{n} z_{n}\right\|\left\|y_{n}-p\right\| \\
\leq & \beta_{n}\left(\left(1+\gamma_{n}\right)\left\|z_{n}-p\right\|^{2}+c_{n}\right)+\left(1-\beta_{n}\right)\left\|z_{n}-p\right\|^{2}-\beta_{n}\left(1-\beta_{n}\right)\left\|k_{n}-W_{n} z_{n}\right\|^{2} \\
& +2 \alpha_{n}\left\|u+\gamma f\left(x_{n}\right)-\bar{V} W_{n} z_{n}\right\|\left\|y_{n}-p\right\| \\
\leq & \beta_{n}\left(\left(1+\gamma_{n}\right)\left\|z_{n}-p\right\|^{2}+c_{n}\right)+\left(1-\beta_{n}\right)\left(\left(1+\gamma_{n}\right)\left\|z_{n}-p\right\|^{2}+c_{n}\right) \\
& -\beta_{n}\left(1-\beta_{n}\right)\left\|k_{n}-W_{n} z_{n}\right\|^{2}+2 \alpha_{n}\left\|u+\gamma f\left(x_{n}\right)-\bar{V} W_{n} z_{n}\right\|\left\|y_{n}-p\right\| \\
= & \left(1+\gamma_{n}\right)\left\|z_{n}-p\right\|^{2}+c_{n}-\beta_{n}\left(1-\beta_{n}\right)\left\|k_{n}-W_{n} z_{n}\right\|^{2} \\
& +2 \alpha_{n}\left\|u+\gamma f\left(x_{n}\right)-\bar{V} W_{n} z_{n}\right\|\left\|y_{n}-p\right\| \\
\leq & \left(1+\gamma_{n}\right)\left\|x_{n}-p\right\|^{2}+c_{n}-\beta_{n}\left(1-\beta_{n}\right)\left\|k_{n}-W_{n} z_{n}\right\|^{2} \\
& +2 \alpha_{n}\left\|u+\gamma f\left(x_{n}\right)-\bar{V} W_{n} z_{n}\right\|\left\|y_{n}-p\right\|,
\end{aligned}
$$

which leads to

$$
\begin{aligned}
\beta_{n}(1 & \left.-\beta_{n}\right)\left\|k_{n}-W_{n} z_{n}\right\|^{2} \\
\leq & \left\|x_{n}-p\right\|^{2}-\left\|y_{n}-p\right\|^{2}+\gamma_{n}\left\|x_{n}-p\right\|^{2}+c_{n} \\
& +2 \alpha_{n}\left\|u+\gamma f\left(x_{n}\right)-\bar{V} W_{n} z_{n}\right\|\left\|y_{n}-p\right\| \\
\leq & \left\|x_{n}-y_{n}\right\|\left(\left\|x_{n}-p\right\|+\left\|y_{n}-p\right\|\right)+\gamma_{n}\left\|x_{n}-p\right\|^{2}+c_{n} \\
& +2 \alpha_{n}\left\|u+\gamma f\left(x_{n}\right)-\bar{V} W_{n} z_{n}\right\|\left\|y_{n}-p\right\| .
\end{aligned}
$$

Since $\lim _{n \rightarrow \infty} \alpha_{n}=0, \lim _{n \rightarrow \infty} \gamma_{n}=0$, and $\lim _{n \rightarrow \infty} c_{n}=0$, it follows from (3.9) and condition (iii) that

$$
\lim _{n \rightarrow \infty}\left\|k_{n}-W_{n} z_{n}\right\|=0
$$

Note that

$$
y_{n}-k_{n}=\alpha_{n}\left(u+\gamma f\left(x_{n}\right)-\bar{V} W_{n} z_{n}\right)+\left(1-\beta_{n}\right)\left(W_{n} z_{n}-k_{n}\right),
$$

which yields

$$
\begin{aligned}
\left\|x_{n}-k_{n}\right\| & \leq\left\|x_{n}-y_{n}\right\|+\left\|y_{n}-k_{n}\right\| \\
& \leq\left\|x_{n}-y_{n}\right\|+\left\|\alpha_{n}\left(u+\gamma f\left(x_{n}\right)-\bar{V} W_{n} z_{n}\right)+\left(1-\beta_{n}\right)\left(W_{n} z_{n}-k_{n}\right)\right\| \\
& \leq\left\|x_{n}-y_{n}\right\|+\alpha_{n}\left\|u+\gamma f\left(x_{n}\right)-\bar{V} W_{n} z_{n}\right\|+\left(1-\beta_{n}\right)\left\|W_{n} z_{n}-k_{n}\right\| \\
& \leq\left\|x_{n}-y_{n}\right\|+\alpha_{n}\left\|u+\gamma f\left(x_{n}\right)-\bar{V} W_{n} z_{n}\right\|+\left\|W_{n} z_{n}-k_{n}\right\| .
\end{aligned}
$$

So, from (3.9), (3.10), and $\lim _{n \rightarrow \infty} \alpha_{n}=0$, we get

$$
\lim _{n \rightarrow \infty}\left\|x_{n}-k_{n}\right\|=0
$$


Step 3. We prove that $\left\|x_{n}-u_{n}\right\| \rightarrow 0,\left\|u_{n}-z_{n}\right\| \rightarrow 0,\left\|z_{n}-W z_{n}\right\| \rightarrow 0$, and $\left\|z_{n}-S^{n} z_{n}\right\| \rightarrow$ 0 as $n \rightarrow \infty$.

Indeed, taking into consideration that $0<\liminf _{n \rightarrow \infty} r_{n} \leq \limsup _{n \rightarrow \infty} r_{n}<2 \zeta$, we may assume, without loss of generality, that $\left\{r_{n}\right\} \subset[c, d] \subset(0,2 \zeta)$. From (3.4) and (3.5) it follows that

$$
\begin{aligned}
\left\|k_{n}-p\right\|^{2} & \leq\left[1+\gamma_{n}\left(1-\delta_{n}\right)\right]\left\|z_{n}-p\right\|^{2}+\left(1-\delta_{n}\right)\left(k-\delta_{n}\right)\left\|z_{n}-S^{n} z_{n}\right\|^{2}+\left(1-\delta_{n}\right) c_{n} \\
& \leq\left\|z_{n}-p\right\|^{2}+\gamma_{n}\left\|z_{n}-p\right\|^{2}+c_{n} \\
& \leq\left\|z_{n}-p\right\|^{2}+\gamma_{n}\left\|x_{n}-p\right\|^{2}+c_{n} .
\end{aligned}
$$

Next we prove that

$$
\lim _{n \rightarrow \infty}\left\|x_{n}-u_{n}\right\|=0
$$

For $p \in \Omega$, we find that

$$
\begin{aligned}
\left\|u_{n}-p\right\|^{2} & =\left\|S_{r_{n}}^{(\Theta, \varphi)}\left(I-r_{n} A\right) x_{n}-S_{r_{n}}^{(\Theta, \varphi)}\left(I-r_{n} A\right) p\right\|^{2} \\
& \leq\left\|\left(I-r_{n} A\right) x_{n}-\left(I-r_{n} A\right) p\right\|^{2} \\
& =\left\|x_{n}-p-r_{n}\left(A x_{n}-A p\right)\right\|^{2} \\
& \leq\left\|x_{n}-p\right\|^{2}+r_{n}\left(r_{n}-2 \zeta\right)\left\|A x_{n}-A p\right\|^{2} .
\end{aligned}
$$

By (3.3), (3.12), and (3.14), we obtain

$$
\begin{aligned}
\left\|k_{n}-p\right\|^{2} & \leq\left\|z_{n}-p\right\|^{2}+\gamma_{n}\left\|x_{n}-p\right\|^{2}+c_{n} \\
& \leq\left\|u_{n}-p\right\|^{2}+\gamma_{n}\left\|x_{n}-p\right\|^{2}+c_{n} \\
& \leq\left\|x_{n}-p\right\|^{2}+r_{n}\left(r_{n}-2 \zeta\right)\left\|A x_{n}-A p\right\|^{2}+\gamma_{n}\left\|x_{n}-p\right\|^{2}+c_{n},
\end{aligned}
$$

which implies that

$$
\begin{aligned}
c(2 \zeta-d)\left\|A x_{n}-A p\right\|^{2} & \leq r_{n}\left(2 \zeta-r_{n}\right)\left\|A x_{n}-A p\right\|^{2} \\
& \leq\left\|x_{n}-p\right\|^{2}-\left\|k_{n}-p\right\|^{2}+\gamma_{n}\left\|x_{n}-p\right\|^{2}+c_{n} \\
& \leq\left\|x_{n}-k_{n}\right\|\left(\left\|x_{n}-p\right\|+\left\|k_{n}-p\right\|\right)+\gamma_{n}\left\|x_{n}-p\right\|^{2}+c_{n} .
\end{aligned}
$$

From $\lim _{n \rightarrow \infty} \gamma_{n}=0, \lim _{n \rightarrow \infty} c_{n}=0$, and (3.11), we have

$$
\lim _{n \rightarrow \infty}\left\|A x_{n}-A p\right\|=0
$$

By the firm nonexpansivity of $S_{r_{n}}^{(\Theta, \varphi)}$ and Lemma 2.2(a), we have

$$
\begin{aligned}
& \left\|u_{n}-p\right\|^{2} \\
& \quad=\left\|S_{r_{n}}^{(\Theta, \varphi)}\left(I-r_{n} A\right) x_{n}-S_{r_{n}}^{(\Theta, \varphi)}\left(I-r_{n} A\right) p\right\|^{2}
\end{aligned}
$$




$$
\begin{aligned}
\leq & \left\langle\left(I-r_{n} A\right) x_{n}-\left(I-r_{n} A\right) p, u_{n}-p\right\rangle \\
= & \frac{1}{2}\left[\left\|\left(I-r_{n} A\right) x_{n}-\left(I-r_{n} A\right) p\right\|^{2}+\left\|u_{n}-p\right\|^{2}\right. \\
& \left.-\left\|\left(I-r_{n} A\right) x_{n}-\left(I-r_{n} A\right) p-\left(u_{n}-p\right)\right\|^{2}\right] \\
\leq & \frac{1}{2}\left[\left\|x_{n}-p\right\|^{2}+\left\|u_{n}-p\right\|^{2}-\left\|x_{n}-u_{n}-r_{n}\left(A x_{n}-A p\right)\right\|^{2}\right] \\
= & \frac{1}{2}\left[\left\|x_{n}-p\right\|^{2}+\left\|u_{n}-p\right\|^{2}-\left\|x_{n}-u_{n}\right\|^{2}+2 r_{n}\left\langle A x_{n}-A p, x_{n}-u_{n}\right\rangle\right. \\
& \left.-r_{n}^{2}\left\|A x_{n}-A p\right\|^{2}\right],
\end{aligned}
$$

which implies that

$$
\left\|u_{n}-p\right\|^{2} \leq\left\|x_{n}-p\right\|^{2}-\left\|x_{n}-u_{n}\right\|^{2}+2 r_{n}\left\|A x_{n}-A p\right\|\left\|x_{n}-u_{n}\right\| .
$$

Combining (3.12) and (3.16), we have

$$
\begin{aligned}
\left\|k_{n}-p\right\|^{2} & \leq\left\|z_{n}-p\right\|^{2}+\gamma_{n}\left\|x_{n}-p\right\|^{2}+c_{n} \\
& \leq\left\|u_{n}-p\right\|^{2}+\gamma_{n}\left\|x_{n}-p\right\|^{2}+c_{n} \\
& \leq\left\|x_{n}-p\right\|^{2}-\left\|x_{n}-u_{n}\right\|^{2}+2 r_{n}\left\|A x_{n}-A p\right\|\left\|x_{n}-u_{n}\right\|+\gamma_{n}\left\|x_{n}-p\right\|^{2}+c_{n},
\end{aligned}
$$

which implies

$$
\begin{aligned}
\| x_{n}- & u_{n} \|^{2} \\
\leq & \left\|x_{n}-p\right\|^{2}-\left\|k_{n}-p\right\|^{2}+2 r_{n}\left\|A x_{n}-A p\right\|\left\|x_{n}-u_{n}\right\|+\gamma_{n}\left\|x_{n}-p\right\|^{2}+c_{n} \\
\leq & \left\|x_{n}-k_{n}\right\|\left(\left\|x_{n}-p\right\|+\left\|k_{n}-p\right\|\right)+2 r_{n}\left\|A x_{n}-A p\right\|\left\|x_{n}-u_{n}\right\| \\
& +\gamma_{n}\left\|x_{n}-p\right\|^{2}+c_{n} .
\end{aligned}
$$

From $\lim _{n \rightarrow \infty} \gamma_{n}=0, \lim _{n \rightarrow \infty} c_{n}=0$, (3.11), and (3.15), we know that (3.13) holds.

Next we show that $\lim _{n \rightarrow \infty}\left\|B_{i} \Lambda_{n}^{i} u_{n}-B_{i} p\right\|=0, i=1,2, \ldots, N$. It follows from Lemma 2.14 that

$$
\begin{aligned}
\left\|\Lambda_{n}^{i} u_{n}-p\right\|^{2} & =\left\|J_{R_{i}, \lambda_{i, n}}\left(I-\lambda_{i, n} B_{i}\right) \Lambda_{n}^{i-1} u_{n}-J_{R_{i}, \lambda_{i, n}}\left(I-\lambda_{i, n} B_{i}\right) p\right\|^{2} \\
& \leq\left\|\left(I-\lambda_{i, n} B_{i}\right) \Lambda_{n}^{i-1} u_{n}-\left(I-\lambda_{i, n} B_{i}\right) p\right\|^{2} \\
& \leq\left\|\Lambda_{n}^{i-1} u_{n}-p\right\|^{2}+\lambda_{i, n}\left(\lambda_{i, n}-2 \eta_{i}\right)\left\|B_{i} \Lambda_{n}^{i-1} u_{n}-B_{i} p\right\|^{2} \\
& \leq\left\|u_{n}-p\right\|^{2}+\lambda_{i, n}\left(\lambda_{i, n}-2 \eta_{i}\right)\left\|B_{i} \Lambda_{n}^{i-1} u_{n}-B_{i} p\right\|^{2} \\
& \leq\left\|x_{n}-p\right\|^{2}+\lambda_{i, n}\left(\lambda_{i, n}-2 \eta_{i}\right)\left\|B_{i} \Lambda_{n}^{i-1} u_{n}-B_{i} p\right\|^{2} .
\end{aligned}
$$

Combining (3.12) and (3.17), we have

$$
\begin{aligned}
\left\|k_{n}-p\right\|^{2} & \leq\left\|z_{n}-p\right\|^{2}+\gamma_{n}\left\|x_{n}-p\right\|^{2}+c_{n} \\
& \leq\left\|\Lambda_{n}^{i} u_{n}-p\right\|^{2}+\gamma_{n}\left\|x_{n}-p\right\|^{2}+c_{n}
\end{aligned}
$$




$$
\begin{aligned}
\leq & \left\|x_{n}-p\right\|^{2}+\lambda_{i, n}\left(\lambda_{i, n}-2 \eta_{i}\right)\left\|B_{i} \Lambda_{n}^{i-1} u_{n}-B_{i} p\right\|^{2} \\
& +\gamma_{n}\left\|x_{n}-p\right\|^{2}+c_{n},
\end{aligned}
$$

together with $\left\{\lambda_{i, n}\right\} \subset\left[a_{i}, b_{i}\right] \subset\left(0,2 \eta_{i}\right), i \in\{1,2, \ldots, N\}$, implies

$$
\begin{aligned}
a_{i}\left(2 \eta_{i}-b_{i}\right)\left\|B_{i} \Lambda_{n}^{i-1} u_{n}-B_{i} p\right\|^{2} \leq & \lambda_{i, n}\left(2 \eta_{i}-\lambda_{i, n}\right)\left\|B_{i} \Lambda_{n}^{i-1} u_{n}-B_{i} p\right\|^{2} \\
\leq & \left\|x_{n}-p\right\|^{2}-\left\|k_{n}-p\right\|^{2}+\gamma_{n}\left\|x_{n}-p\right\|^{2}+c_{n} \\
\leq & \left\|x_{n}-k_{n}\right\|\left(\left\|x_{n}-p\right\|+\left\|k_{n}-p\right\|\right) \\
& +\gamma_{n}\left\|x_{n}-p\right\|^{2}+c_{n} .
\end{aligned}
$$

From $\lim _{n \rightarrow \infty} \gamma_{n}=0, \lim _{n \rightarrow \infty} c_{n}=0$, and (3.11), we obtain

$$
\lim _{n \rightarrow \infty}\left\|B_{i} \Lambda_{n}^{i-1} u_{n}-B_{i} p\right\|=0, \quad i=1,2, \ldots, N
$$

By Lemma 2.14 and Lemma 2.2(a), we obtain

$$
\begin{aligned}
\left\|\Lambda_{n}^{i} u_{n}-p\right\|^{2}= & \left\|J_{R_{i}, \lambda_{i, n}}\left(I-\lambda_{i, n} B_{i}\right) \Lambda_{n}^{i-1} u_{n}-J_{R_{i}, \lambda_{i, n}}\left(I-\lambda_{i, n} B_{i}\right) p\right\|^{2} \\
\leq & \left\langle\left(I-\lambda_{i, n} B_{i}\right) \Lambda_{n}^{i-1} u_{n}-\left(I-\lambda_{i, n} B_{i}\right) p, \Lambda_{n}^{i} u_{n}-p\right\rangle \\
= & \frac{1}{2}\left(\left\|\left(I-\lambda_{i, n} B_{i}\right) \Lambda_{n}^{i-1} u_{n}-\left(I-\lambda_{i, n} B_{i}\right) p\right\|^{2}+\left\|\Lambda_{n}^{i} u_{n}-p\right\|^{2}\right. \\
& \left.-\left\|\left(I-\lambda_{i, n} B_{i}\right) \Lambda_{n}^{i-1} u_{n}-\left(I-\lambda_{i, n} B_{i}\right) p-\left(\Lambda_{n}^{i} u_{n}-p\right)\right\|^{2}\right) \\
\leq & \frac{1}{2}\left(\left\|\Lambda_{n}^{i-1} u_{n}-p\right\|^{2}+\left\|\Lambda_{n}^{i} u_{n}-p\right\|^{2}\right. \\
& \left.-\left\|\Lambda_{n}^{i-1} u_{n}-\Lambda_{n}^{i} u_{n}-\lambda_{i, n}\left(B_{i} \Lambda_{n}^{i-1} u_{n}-B_{i} p\right)\right\|^{2}\right) \\
\leq & \frac{1}{2}\left(\left\|u_{n}-p\right\|^{2}+\left\|\Lambda_{n}^{i} u_{n}-p\right\|^{2}\right. \\
& \left.-\left\|\Lambda_{n}^{i-1} u_{n}-\Lambda_{n}^{i} u_{n}-\lambda_{i, n}\left(B_{i} \Lambda_{n}^{i-1} u_{n}-B_{i} p\right)\right\|^{2}\right) \\
\leq & \frac{1}{2}\left(\left\|x_{n}-p\right\|^{2}+\left\|\Lambda_{n}^{i} u_{n}-p\right\|^{2}\right. \\
& \left.-\left\|\Lambda_{n}^{i-1} u_{n}-\Lambda_{n}^{i} u_{n}-\lambda_{i, n}\left(B_{i} \Lambda_{n}^{i-1} u_{n}-B_{i} p\right)\right\|^{2}\right),
\end{aligned}
$$

which implies

$$
\begin{aligned}
&\left\|\Lambda_{n}^{i} u_{n}-p\right\|^{2} \\
& \leq\left\|x_{n}-p\right\|^{2}-\left\|\Lambda_{n}^{i-1} u_{n}-\Lambda_{n}^{i} u_{n}-\lambda_{i, n}\left(B_{i} \Lambda_{n}^{i-1} u_{n}-B_{i} p\right)\right\|^{2} \\
&=\left\|x_{n}-p\right\|^{2}-\left\|\Lambda_{n}^{i-1} u_{n}-\Lambda_{n}^{i} u_{n}\right\|^{2}-\lambda_{i, n}^{2}\left\|B_{i} \Lambda_{n}^{i-1} u_{n}-B_{i} p\right\|^{2} \\
&+2 \lambda_{i, n}\left\langle\Lambda_{n}^{i-1} u_{n}-\Lambda_{n}^{i} u_{n}, B_{i} \Lambda_{n}^{i-1} u_{n}-B_{i} p\right\rangle \\
& \leq\left\|x_{n}-p\right\|^{2}-\left\|\Lambda_{n}^{i-1} u_{n}-\Lambda_{n}^{i} u_{n}\right\|^{2} \\
&+2 \lambda_{i, n}\left\|\Lambda_{n}^{i-1} u_{n}-\Lambda_{n}^{i} u_{n}\right\|\left\|B_{i} \Lambda_{n}^{i-1} u_{n}-B_{i} p\right\| .
\end{aligned}
$$


Combining (3.12) and (3.19) we get

$$
\begin{aligned}
\left\|k_{n}-p\right\|^{2} \leq & \left\|z_{n}-p\right\|^{2}+\gamma_{n}\left\|x_{n}-p\right\|^{2}+c_{n} \\
\leq & \left\|\Lambda_{n}^{i} u_{n}-p\right\|^{2}+\gamma_{n}\left\|x_{n}-p\right\|^{2}+c_{n} \\
\leq & \left\|x_{n}-p\right\|^{2}-\left\|\Lambda_{n}^{i-1} u_{n}-\Lambda_{n}^{i} u_{n}\right\|^{2} \\
& +2 \lambda_{i, n}\left\|\Lambda_{n}^{i-1} u_{n}-\Lambda_{n}^{i} u_{n}\right\|\left\|B_{i} \Lambda_{n}^{i-1} u_{n}-B_{i} p\right\| \\
& +\gamma_{n}\left\|x_{n}-p\right\|^{2}+c_{n},
\end{aligned}
$$

which implies

$$
\begin{aligned}
& \left\|\Lambda_{n}^{i-1} u_{n}-\Lambda_{n}^{i} u_{n}\right\|^{2} \\
& \leq\left\|x_{n}-p\right\|^{2}-\left\|k_{n}-p\right\|^{2}+2 \lambda_{i, n}\left\|\Lambda_{n}^{i-1} u_{n}-\Lambda_{n}^{i} u_{n}\right\|\left\|B_{i} \Lambda_{n}^{i-1} u_{n}-B_{i} p\right\| \\
& \quad+\gamma_{n}\left\|x_{n}-p\right\|^{2}+c_{n} \\
& \leq\left\|x_{n}-k_{n}\right\|\left(\left\|x_{n}-p\right\|+\left\|k_{n}-p\right\|\right)+2 \lambda_{i, n}\left\|\Lambda_{n}^{i-1} u_{n}-\Lambda_{n}^{i} u_{n}\right\|\left\|B_{i} \Lambda_{n}^{i-1} u_{n}-B_{i} p\right\| \\
& \quad+\gamma_{n}\left\|x_{n}-p\right\|^{2}+c_{n} .
\end{aligned}
$$

From (3.11), (3.18), $\lim _{n \rightarrow \infty} \gamma_{n}=0$, and $\lim _{n \rightarrow \infty} c_{n}=0$, we have

$$
\lim _{n \rightarrow \infty}\left\|\Lambda_{n}^{i-1} u_{n}-\Lambda_{n}^{i} u_{n}\right\|=0, \quad i=1,2, \ldots, N
$$

From (3.20) we get

$$
\begin{aligned}
\left\|u_{n}-z_{n}\right\| & =\left\|\Lambda_{n}^{0} u_{n}-\Lambda_{n}^{N} u_{n}\right\| \\
& \leq\left\|\Lambda_{n}^{0} u_{n}-\Lambda_{n}^{1} u_{n}\right\|+\left\|\Lambda_{n}^{1} u_{n}-\Lambda_{n}^{2} u_{n}\right\|+\cdots+\left\|\Lambda_{n}^{N-1} u_{n}-\Lambda_{n}^{N} u_{n}\right\| \\
& \rightarrow 0 \quad \text { as } n \rightarrow \infty
\end{aligned}
$$

By (3.13) and (3.21), we have

$$
\begin{aligned}
\left\|x_{n}-z_{n}\right\| & \leq\left\|x_{n}-u_{n}\right\|+\left\|u_{n}-z_{n}\right\| \\
& \rightarrow 0 \quad \text { as } n \rightarrow \infty
\end{aligned}
$$

From (3.8) and (3.22), we have

$$
\begin{aligned}
\left\|z_{n+1}-z_{n}\right\| & \leq\left\|z_{n+1}-x_{n+1}\right\|+\left\|x_{n+1}-x_{n}\right\|+\left\|x_{n}-z_{n}\right\| \\
& \rightarrow 0 \quad \text { as } n \rightarrow \infty .
\end{aligned}
$$

By (3.11), (3.13), and (3.21), we get

$$
\begin{aligned}
\left\|k_{n}-z_{n}\right\| & \leq\left\|k_{n}-x_{n}\right\|+\left\|x_{n}-u_{n}\right\|+\left\|u_{n}-z_{n}\right\| \\
& \rightarrow 0 \quad \text { as } n \rightarrow \infty .
\end{aligned}
$$


We observe that

$$
k_{n}-z_{n}=\left(1-\delta_{n}\right)\left(S^{n} z_{n}-z_{n}\right) .
$$

From $\delta_{n} \leq d<1$ and (3.24), we have

$$
\lim _{n \rightarrow \infty}\left\|S^{n} z_{n}-z_{n}\right\|=0
$$

We note that

$$
\begin{aligned}
\left\|S^{n} z_{n}-S^{n+1} z_{n}\right\| \leq & \left\|S^{n} z_{n}-z_{n}\right\|+\left\|z_{n}-z_{n+1}\right\|+\left\|z_{n+1}-S^{n+1} z_{n+1}\right\| \\
& +\left\|S^{n+1} z_{n+1}-S^{n+1} z_{n}\right\| .
\end{aligned}
$$

From (3.23), (3.25), and Lemma 2.4, we obtain

$$
\lim _{n \rightarrow \infty}\left\|S^{n} z_{n}-S^{n+1} z_{n}\right\|=0 .
$$

On the other hand, we note that

$$
\left\|z_{n}-S z_{n}\right\| \leq\left\|z_{n}-S^{n} z_{n}\right\|+\left\|S^{n} z_{n}-S^{n+1} z_{n}\right\|+\left\|S^{n+1} z_{n}-S z_{n}\right\| .
$$

From (3.25), (3.26), and the uniform continuity of $S$, we have

$$
\lim _{n \rightarrow \infty}\left\|z_{n}-S z_{n}\right\|=0
$$

In addition, note that

$$
\left\|z_{n}-W z_{n}\right\| \leq\left\|z_{n}-k_{n}\right\|+\left\|k_{n}-W_{n} z_{n}\right\|+\left\|W_{n} z_{n}-W z_{n}\right\| .
$$

So, from (3.10), (3.24), and Remark 2.3 it follows that

$$
\lim _{n \rightarrow \infty}\left\|z_{n}-W z_{n}\right\|=0
$$

Step 4. we prove that $x_{n} \rightarrow v=P_{\Omega} x_{0}$ as $n \rightarrow \infty$.

Indeed, since $\left\{x_{n}\right\}$ is bounded, there exists a subsequence $\left\{x_{n_{i}}\right\}$ which converges weakly to some $w$. From (3.13) and (3.20)-(3.22), we see that $u_{n_{i}} \rightarrow w, \Lambda_{n_{i}}^{m} u_{n_{i}} \rightarrow w$, and $z_{n_{i}} \rightarrow w$, where $m \in\{1,2, \ldots, N\}$. Since $S$ is uniformly continuous, by (3.27) we get $\lim _{n \rightarrow \infty} \| z_{n}-$ $S^{m} z_{n} \|=0$ for any $m \geq 1$. Hence from Lemma 2.6, we obtain $w \in \operatorname{Fix}(S)$. In the meantime, utilizing Lemma 2.13, we deduce from (3.28) and $z_{n_{i}} \rightarrow w$ that $w \in \operatorname{Fix}(W)=\bigcap_{n=1}^{\infty} \operatorname{Fix}\left(T_{n}\right)$ (due to Lemma 2.12). Next, we prove that $w \in \bigcap_{m=1}^{N} \mathrm{I}\left(B_{m}, R_{m}\right)$. As a matter of fact, since $B_{m}$ is $\eta_{m}$-inverse-strongly monotone, $B_{m}$ is a monotone and Lipschitz-continuous mapping. It follows from Lemma 2.17 that $R_{m}+B_{m}$ is maximal monotone. Let $(v, g) \in G\left(R_{m}+B_{m}\right)$, i.e., $g-B_{m} v \in R_{m} v$. Again, since $\Lambda_{n}^{m} u_{n}=J_{R_{m}, \lambda_{m, n}}\left(I-\lambda_{m, n} B_{m}\right) \Lambda_{n}^{m-1} u_{n}, n \geq 1, m \in\{1,2, \ldots, N\}$, we have

$$
\Lambda_{n}^{m-1} u_{n}-\lambda_{m, n} B_{m} \Lambda_{n}^{m-1} u_{n} \in\left(I+\lambda_{m, n} R_{m}\right) \Lambda_{n}^{m} u_{n},
$$


that is,

$$
\frac{1}{\lambda_{m, n}}\left(\Lambda_{n}^{m-1} u_{n}-\Lambda_{n}^{m} u_{n}-\lambda_{m, n} B_{m} \Lambda_{n}^{m-1} u_{n}\right) \in R_{m} \Lambda_{n}^{m} u_{n} .
$$

In terms of the monotonicity of $R_{m}$, we get

$$
\left\langle v-\Lambda_{n}^{m} u_{n}, g-B_{m} v-\frac{1}{\lambda_{m, n}}\left(\Lambda_{n}^{m-1} u_{n}-\Lambda_{n}^{m} u_{n}-\lambda_{m, n} B_{m} \Lambda_{n}^{m-1} u_{n}\right)\right\rangle \geq 0
$$

and hence

$$
\begin{aligned}
\langle v- & \left.\Lambda_{n}^{m} u_{n}, g\right\rangle \\
& \geq\left\langle v-\Lambda_{n}^{m} u_{n}, B_{m} v+\frac{1}{\lambda_{m, n}}\left(\Lambda_{n}^{m-1} u_{n}-\Lambda_{n}^{m} u_{n}-\lambda_{m, n} B_{m} \Lambda_{n}^{m-1} u_{n}\right)\right\rangle \\
& =\left\langle v-\Lambda_{n}^{m} u_{n}, B_{m} v-B_{m} \Lambda_{n}^{m} u_{n}+B_{m} \Lambda_{n}^{m} u_{n}-B_{m} \Lambda_{n}^{m-1} u_{n}+\frac{1}{\lambda_{m, n}}\left(\Lambda_{n}^{m-1} u_{n}-\Lambda_{n}^{m} u_{n}\right)\right\rangle \\
& \geq\left\langle v-\Lambda_{n}^{m} u_{n}, B_{m} \Lambda_{n}^{m} u_{n}-B_{m} \Lambda_{n}^{m-1} u_{n}\right\rangle+\left\langle v-\Lambda_{n}^{m} u_{n}, \frac{1}{\lambda_{m, n}}\left(\Lambda_{n}^{m-1} u_{n}-\Lambda_{n}^{m} u_{n}\right)\right\rangle .
\end{aligned}
$$

In particular,

$$
\begin{aligned}
\left\langle v-\Lambda_{n_{i}}^{m} u_{n_{i}}, g\right\rangle \geq & \left\langle v-\Lambda_{n_{i}}^{m} u_{n_{i}}, B_{m} \Lambda_{n_{i}}^{m} u_{n_{i}}-B_{m} \Lambda_{n_{i}}^{m-1} u_{n_{i}}\right\rangle \\
& +\left\langle v-\Lambda_{n_{i}}^{m} u_{n_{i}}, \frac{1}{\lambda_{m, n_{i}}}\left(\Lambda_{n_{i}}^{m-1} u_{n_{i}}-\Lambda_{n_{i}}^{m} u_{n_{i}}\right)\right\rangle .
\end{aligned}
$$

Since $\left\|\Lambda_{n}^{m} u_{n}-\Lambda_{n}^{m-1} u_{n}\right\| \rightarrow 0$ (due to (3.20)) and $\left\|B_{m} \Lambda_{n}^{m} u_{n}-B_{m} \Lambda_{n}^{m-1} u_{n}\right\| \rightarrow 0$ (due to the Lipschitz-continuity of $\left.B_{m}\right)$, we conclude from $\Lambda_{n_{i}}^{m} u_{n_{i}} \rightarrow w$ and $\left\{\lambda_{i, n}\right\} \subset\left[a_{i}, b_{i}\right] \subset\left(0,2 \eta_{i}\right)$, $i \in\{1,2, \ldots, N\}$ that

$$
\lim _{i \rightarrow \infty}\left\langle v-\Lambda_{n_{i}}^{m} u_{n_{i}}, g\right\rangle=\langle v-w, g\rangle \geq 0 .
$$

It follows from the maximal monotonicity of $B_{m}+R_{m}$ that $0 \in\left(R_{m}+B_{m}\right) w$, i.e., $w \in$ $\mathrm{I}\left(B_{m}, R_{m}\right)$. Therefore, $w \in \bigcap_{m=1}^{N} \mathrm{I}\left(B_{m}, R_{m}\right)$.

Next, we show that $w \in \operatorname{GMEP}(\Theta, \varphi, A)$. In fact, from $z_{n}=S_{r_{n}}^{(\Theta, \varphi)}\left(I-r_{n} A\right) x_{n}$, we know that

$$
\Theta\left(u_{n}, y\right)+\varphi(y)-\varphi\left(u_{n}\right)+\left\langle A x_{n}, y-u_{n}\right\rangle+\frac{1}{r_{n}}\left\langle K^{\prime}\left(u_{n}\right)-K^{\prime}\left(x_{n}\right), y-u_{n}\right\rangle \geq 0, \quad \forall y \in C .
$$

From (H2) it follows that

$$
\varphi(y)-\varphi\left(u_{n}\right)+\left\langle A x_{n}, y-u_{n}\right\rangle+\frac{1}{r_{n}}\left\langle K^{\prime}\left(u_{n}\right)-K^{\prime}\left(x_{n}\right), y-u_{n}\right\rangle \geq \Theta\left(y, u_{n}\right), \quad \forall y \in C .
$$

Replacing $n$ by $n_{i}$, we have

$$
\varphi(y)-\varphi\left(u_{n_{i}}\right)+\left\langle A x_{n_{i}}, y-u_{n_{i}}\right\rangle+\left\langle\frac{K^{\prime}\left(u_{n_{i}}\right)-K^{\prime}\left(x_{n_{i}}\right)}{r_{n_{i}}}, y-u_{n_{i}}\right\rangle \geq \Theta\left(y, u_{n_{i}}\right),
$$

$\forall y \in C$. 
Put $u_{t}=t y+(1-t) w$ for all $t \in(0,1]$ and $y \in C$. Then, from (3.29), we have

$$
\begin{aligned}
\left\langle u_{t}-\right. & \left.u_{n_{i}}, A u_{t}\right\rangle \\
\geq & \left\langle u_{t}-u_{n_{i}}, A u_{t}\right\rangle-\varphi\left(u_{t}\right)+\varphi\left(u_{n_{i}}\right)-\left\langle u_{t}-u_{n_{i}}, A x_{n_{i}}\right\rangle \\
& -\left\langle\frac{K^{\prime}\left(u_{n_{i}}\right)-K^{\prime}\left(x_{n_{i}}\right)}{r_{n_{i}}}, u_{t}-u_{n_{i}}\right\rangle+\Theta\left(u_{t}, u_{n_{i}}\right) \\
\geq & \left\langle u_{t}-u_{n_{i}}, A u_{t}-A u_{n_{i}}\right\rangle+\left\langle u_{t}-u_{n_{i}}, A u_{n_{i}}-A x_{n_{i}}\right\rangle-\varphi\left(u_{t}\right)+\varphi\left(u_{n_{i}}\right) \\
& -\left\langle\frac{K^{\prime}\left(u_{n_{i}}\right)-K^{\prime}\left(x_{n_{i}}\right)}{r_{n_{i}}}, u_{t}-u_{n_{i}}\right\rangle+\Theta\left(u_{t}, u_{n_{i}}\right) .
\end{aligned}
$$

Since $\left\|u_{n_{i}}-x_{n_{i}}\right\| \rightarrow 0$ as $i \rightarrow \infty$, we deduce from the Lipschitz-continuity of $A$ and $K^{\prime}$ that $\left\|A u_{n_{i}}-A x_{n_{i}}\right\| \rightarrow 0$ and $\left\|K^{\prime}\left(u_{n_{i}}\right)-K^{\prime}\left(x_{n_{i}}\right)\right\| \rightarrow 0$ as $i \rightarrow \infty$. Further, from the monotonicity of $A$, we have $\left\langle u_{t}-u_{n_{i}}, A u_{t}-A u_{n_{i}}\right\rangle \geq 0$. So, from (H4), we have the weakly lower semicontinuity of $\varphi, \frac{K^{\prime}\left(u_{n_{i}}\right)-K^{\prime}\left(x_{n_{i}}\right)}{r_{n_{i}}} \rightarrow 0$ and $u_{n_{i}} \rightarrow w$, then we have

$$
\left\langle u_{t}-w, A u_{t}\right\rangle \geq-\varphi\left(u_{t}\right)+\varphi(w)+\Theta\left(u_{t}, w\right), \quad \text { as } i \rightarrow \infty
$$

From (H1), (H4), and (3.30) we also have

$$
\begin{aligned}
0 & =\Theta\left(u_{t}, u_{t}\right)+\varphi\left(u_{t}\right)-\varphi\left(u_{t}\right) \\
& \leq t \Theta\left(u_{t}, y\right)+(1-t) \Theta\left(u_{t}, w\right)+t \varphi(y)+(1-t) \varphi(w)-\varphi\left(u_{t}\right) \\
& =t\left[\Theta\left(u_{t}, y\right)+\varphi(y)-\varphi\left(u_{t}\right)\right]+(1-t)\left[\Theta\left(u_{t}, w\right)+\varphi(w)-\varphi(w)-\varphi\left(u_{t}\right)\right] \\
& \leq t\left[\Theta\left(u_{t}, y\right)+\varphi(y)-\varphi\left(u_{t}\right)\right]+(1-t)\left\langle u_{t}-w, A u_{t}\right\rangle \\
& =t\left[\Theta\left(u_{t}, y\right)+\varphi(y)-\varphi\left(u_{t}\right)\right]+(1-t) t\left\langle y-w, A u_{t}\right\rangle
\end{aligned}
$$

and hence

$$
0 \leq \Theta\left(u_{t}, y\right)+\varphi(y)-\varphi\left(u_{t}\right)+(1-t)\left\langle y-w, A u_{t}\right\rangle
$$

Letting $t \rightarrow 0$, we have, for each $y \in C$,

$$
0 \leq \Theta(w, y)+\varphi(y)-\varphi(w)+\langle A w, y-w\rangle
$$

This implies that $w \in \operatorname{GMEP}(\Theta, \varphi, A)$. Therefore,

$$
w \in \bigcap_{n=1}^{\infty} \operatorname{Fix}\left(T_{n}\right) \cap \operatorname{GMEP}(\Theta, \varphi, A) \cap\left(\bigcap_{i=1}^{N} \mathrm{I}\left(B_{i}, R_{i}\right)\right) \cap \operatorname{Fix}(S):=\Omega .
$$

This shows that $\omega_{w}\left(x_{n}\right) \subset \Omega$. From (3.7) and Lemma 2.10 we infer that $x_{n} \rightarrow v=P_{\Omega} x_{0}$ as $n \rightarrow \infty$.

Finally, assume additionally that $\gamma_{n}+c_{n}+\left\|x_{n}-y_{n}\right\|=o\left(\alpha_{n}\right)$. Note that $V$ is a $\bar{\gamma}$-strongly positive bounded linear operator and $f: H \rightarrow H$ is an $l$-Lipschitzian mapping with $\gamma l<$ $(1+\mu) \bar{\gamma}$. It is clear that

$$
\langle(\bar{V} x-(u+\gamma f(x)))-(\bar{V} y-(u+\gamma f(y))), x-y\rangle \geq((1+\mu) \bar{\gamma}-\gamma l)\|x-y\|^{2}, \quad \forall x, y \in H .
$$


Hence we deduce that $\bar{V} x-(u+\gamma f(x))$ is $((1+\mu) \bar{\gamma}-\gamma l)$-strongly monotone. In the meantime, it is easy to see that $\bar{V} x-(u+\gamma f(x))$ is $(\|\bar{V}\|+\gamma l)$-Lipschitzian with constant $\|\bar{V}\|+\gamma l>0$. Thus, there exists a unique solution $p$ in $\Omega$ to the VIP

$$
\langle\bar{V} p-(u+\gamma f(p)), u-p\rangle \geq 0, \quad \forall u \in \Omega .
$$

Equivalently, $p \in \Omega$ solves (OP2) (due to Lemma 2.18). Consequently, we deduce from (3.9) and $x_{n} \rightarrow v=P_{\Omega} x_{0}(n \rightarrow \infty)$ that

$$
\begin{aligned}
& \limsup _{n \rightarrow \infty}\left\langle(u+\gamma f(p))-\bar{V} p, y_{n}-p\right\rangle \\
& \quad=\limsup _{n \rightarrow \infty}\left(\left\langle(u+\gamma f(p))-\bar{V} p, x_{n}-p\right\rangle+\left\langle(u+\gamma f(p))-\bar{V} p, y_{n}-x_{n}\right\rangle\right) \\
& \quad=\limsup _{n \rightarrow \infty}\left\{(u+\gamma f(p))-\bar{V} p, x_{n}-p\right\rangle \\
& \quad=\langle(u+\gamma f(p))-\bar{V} p, v-p\rangle \leq 0 .
\end{aligned}
$$

Furthermore, by Lemma 2.1 we conclude from (3.1), (3.4), and (3.5) that

$$
\begin{aligned}
\| y_{n}- & p \|^{2} \\
= & \| \alpha_{n}(u+\gamma f(p)-\bar{V} p)+\alpha_{n} \gamma\left(f\left(x_{n}\right)-f(p)\right)+\beta_{n}\left(k_{n}-p\right) \\
& +\left(\left(1-\beta_{n}\right) I-\alpha_{n} \bar{V}\right)\left(W_{n} z_{n}-p\right) \|^{2} \\
\leq & \left\|\alpha_{n} \gamma\left(f\left(x_{n}\right)-f(p)\right)+\beta_{n}\left(k_{n}-p\right)+\left(\left(1-\beta_{n}\right) I-\alpha_{n} \bar{V}\right)\left(W_{n} z_{n}-p\right)\right\|^{2} \\
& +2 \alpha_{n}\left((u+\gamma f(p)-\bar{V} p), y_{n}-p\right\rangle \\
\leq & {\left[\alpha_{n} \gamma\left\|f\left(x_{n}\right)-f(p)\right\|+\beta_{n}\left\|k_{n}-p\right\|+\left\|\left(\left(1-\beta_{n}\right) I-\alpha_{n} \bar{V}\right)\left(W_{n} z_{n}-p\right)\right\|\right]^{2} } \\
& +2 \alpha_{n}\left((u+\gamma f(p)-\bar{V} p), y_{n}-p\right\rangle \\
\leq & {\left[\alpha_{n} \gamma l\left\|x_{n}-p\right\|+\beta_{n}\left\|k_{n}-p\right\|+\left(1-\beta_{n}-\alpha_{n}-\alpha_{n} \mu \bar{\gamma}\right)\left\|W_{n} z_{n}-p\right\|\right]^{2} } \\
& +2 \alpha_{n}\left((u+\gamma f(p)-\bar{V} p), y_{n}-p\right\rangle \\
\leq & {\left[\alpha_{n} \gamma l\left\|x_{n}-p\right\|+\beta_{n}\left\|k_{n}-p\right\|+\left(1-\beta_{n}-\alpha_{n}(1+\mu) \bar{\gamma}\right)\left\|z_{n}-p\right\|\right]^{2} } \\
& +2 \alpha_{n}\left((u+\gamma f(p)-\bar{V} p), y_{n}-p\right\rangle \\
= & {\left[\alpha_{n}(1+\mu) \bar{\gamma} \cdot \frac{\gamma l}{(1+\mu) \bar{\gamma}}\left\|x_{n}-p\right\|+\beta_{n}\left\|k_{n}-p\right\|\right.} \\
& \left.+\left(1-\beta_{n}-\alpha_{n}(1+\mu) \bar{\gamma}\right)\left\|z_{n}-p\right\|\right]^{2} \\
& +2 \alpha_{n}\left|(u+\gamma f(p)-\bar{V} p), y_{n}-p\right\rangle \\
\leq & \alpha_{n}(1+\mu) \bar{\gamma} \cdot \frac{(\gamma l)^{2}}{(1+\mu)^{2} \bar{\gamma}{ }^{2}}\left\|x_{n}-p\right\|^{2}+\beta_{n}\left\|k_{n}-p\right\|^{2} \\
& +\left(1-\beta_{n}-\alpha_{n}(1+\mu) \bar{\gamma}\right)\left\|z_{n}-p\right\|^{2}+2 \alpha_{n}\left\langle(u+\gamma f(p)-\bar{V} p), y_{n}-p\right\rangle \\
\leq & \alpha_{n}(1+\mu) \bar{\gamma} \cdot \frac{(\gamma l)^{2}}{(1+\mu)^{2} \bar{\gamma}^{2}}\left\|x_{n}-p\right\|^{2}+\beta_{n}\left(\left(1+\gamma_{n}\right)\left\|z_{n}-p\right\|^{2}+c_{n}\right) \\
&
\end{aligned}
$$




$$
\begin{aligned}
& +\left(1-\beta_{n}-\alpha_{n}(1+\mu) \bar{\gamma}\right)\left\|z_{n}-p\right\|^{2}+2 \alpha_{n}\left\langle(u+\gamma f(p)-\bar{V} p), y_{n}-p\right\rangle \\
\leq & \alpha_{n} \frac{(\gamma l)^{2}}{(1+\mu) \bar{\gamma}}\left\|x_{n}-p\right\|^{2}+\beta_{n}\left(\left(1+\gamma_{n}\right)\left\|z_{n}-p\right\|^{2}+c_{n}\right) \\
& +\left(1-\beta_{n}-\alpha_{n}(1+\mu) \bar{\gamma}\right)\left(\left(1+\gamma_{n}\right)\left\|z_{n}-p\right\|^{2}+c_{n}\right) \\
& +2 \alpha_{n}\left\langle(u+\gamma f(p)-\bar{V} p), y_{n}-p\right\rangle \\
= & \alpha_{n} \frac{(\gamma l)^{2}}{(1+\mu) \bar{\gamma}}\left\|x_{n}-p\right\|^{2}+\left(1-\alpha_{n}(1+\mu) \bar{\gamma}\right)\left(\left(1+\gamma_{n}\right)\left\|z_{n}-p\right\|^{2}+c_{n}\right) \\
& +2 \alpha_{n}\left\langle(u+\gamma f(p)-\bar{V} p), y_{n}-p\right\rangle \\
\leq & \alpha_{n} \frac{(\gamma l)^{2}}{(1+\mu) \bar{\gamma}}\left(\left(1+\gamma_{n}\right)\left\|x_{n}-p\right\|^{2}+c_{n}\right) \\
& +\left(1-\alpha_{n}(1+\mu) \bar{\gamma}\right)\left(\left(1+\gamma_{n}\right)\left\|x_{n}-p\right\|^{2}+c_{n}\right)+2 \alpha_{n}\left\langle(u+\gamma f(p)-\bar{V} p), y_{n}-p\right\rangle \\
= & \left(1-\alpha_{n} \frac{(1+\mu)^{2} \bar{\gamma}^{2}-(\gamma l)^{2}}{(1+\mu) \bar{\gamma}}\right)\left(\left(1+\gamma_{n}\right)\left\|x_{n}-p\right\|^{2}+c_{n}\right) \\
& +2 \alpha_{n}\left\langle(u+\gamma f(p)-\bar{V} p), y_{n}-p\right\rangle \\
\leq & \left(1-\alpha_{n} \frac{(1+\mu)^{2} \bar{\gamma}-(\gamma l)^{2}}{(1+\mu) \bar{\gamma}}\right)\left\|x_{n}-p\right\|^{2}+\gamma_{n}\left\|x_{n}-p\right\|^{2}+c_{n} \\
& +2 \alpha_{n}\left|(u+\gamma f(p)-\bar{V} p), y_{n}-p\right\rangle,
\end{aligned}
$$

which hence yields

$$
\begin{aligned}
& \frac{(1+\mu)^{2} \bar{\gamma}^{2}-(\gamma l)^{2}}{(1+\mu) \bar{\gamma}}\left\|x_{n}-p\right\|^{2} \\
& \leq \frac{\left\|x_{n}-p\right\|^{2}-\left\|y_{n}-p\right\|^{2}}{\alpha_{n}}+\frac{\gamma_{n}\left\|x_{n}-p\right\|^{2}+c_{n}}{\alpha_{n}}+2\left\langle(u+\gamma f(p)-\bar{V} p), y_{n}-p\right\rangle \\
& \leq \frac{\left\|x_{n}-y_{n}\right\|}{\alpha_{n}}\left(\left\|x_{n}-p\right\|+\left\|y_{n}-p\right\|\right)+\frac{\gamma_{n}+c_{n}}{\alpha_{n}}\left(\left\|x_{n}-p\right\|^{2}+1\right) \\
& \quad+2\left\langle(u+\gamma f(p)-\bar{V} p), y_{n}-p\right\rangle .
\end{aligned}
$$

Since $\gamma_{n}+c_{n}=o\left(\alpha_{n}\right),\left\|x_{n}-y_{n}\right\|=o\left(\alpha_{n}\right)$, and $x_{n} \rightarrow v=P_{\Omega} x_{0}$, we infer from (3.31) and $0 \leq$ $\gamma l<(1+\mu) \bar{\gamma}$ that as $n \rightarrow \infty$

$$
\frac{(1+\mu)^{2} \bar{\gamma}^{2}-(\gamma l)^{2}}{(1+\mu) \bar{\gamma}}\|v-p\|^{2} \leq 0 .
$$

That is, $p=v=P_{\Omega} x_{0}$. This completes the proof.

Corollary 3.1 Let $C$ be a nonempty closed convex subset of a real Hilbert space H. Let $\Theta$ be a bifunction from $C \times C$ to $\mathbf{R}$ satisfying $(\mathrm{H} 1)-(\mathrm{H} 4)$ and $\varphi: C \rightarrow \mathbf{R}$ be a lower semicontinuous and convex functional. Let $R_{i}: C \rightarrow 2^{H}$ be a maximal monotone mapping and let $A: H \rightarrow H$ and $B_{i}: C \rightarrow H$ be $\zeta$-inverse strongly monotone and $\eta_{i}$-inversestrongly monotone, respectively, for $i=1,2$. Let $S: C \rightarrow C$ be a uniformly continuous asymptotically $\kappa$-strict pseudocontractive mapping in the intermediate sense for some $0 \leq \kappa<1$ with sequence $\left\{\gamma_{n}\right\} \subset[0, \infty)$ such that $\lim _{n \rightarrow \infty} \gamma_{n}=0$ and $\left\{c_{n}\right\} \subset[0, \infty)$ such 
that $\lim _{n \rightarrow \infty} c_{n}=0$. Let $\left\{T_{n}\right\}_{n=1}^{\infty}$ be a sequence of nonexpansive self-mappings on $C$ and $\left\{\lambda_{n}\right\}$ be a sequence in $(0, b]$ for some $b \in(0,1)$. Let $V$ be a $\bar{\gamma}$-strongly positive bounded linear operator and $f: H \rightarrow H$ be an l-Lipschitzian mapping with $\gamma l<(1+\mu) \bar{\gamma}$. Assume that $\Omega:=\left(\bigcap_{n=1}^{\infty} \operatorname{Fix}\left(T_{n}\right)\right) \cap \operatorname{GMEP}(\Theta, \varphi, A) \cap \mathrm{I}\left(B_{2}, R_{2}\right) \cap \mathrm{I}\left(B_{1}, R_{1}\right) \cap \operatorname{Fix}(S)$ is nonempty and bounded. Let $W_{n}$ be the $W$-mapping defined by (1.4) and $\left\{\alpha_{n}\right\},\left\{\beta_{n}\right\}$, and $\left\{\delta_{n}\right\}$ be three sequences in $(0,1)$ such that $\lim _{n \rightarrow \infty} \alpha_{n}=0$ and $\kappa \leq \delta_{n} \leq d<1$. Assume that:

(i) $K: H \rightarrow \mathbf{R}$ is strongly convex with constant $\sigma>0$ and its derivative $K^{\prime}$ is Lipschitz-continuous with constant $v>0$ such that the function $x \mapsto\left\langle y-x, K^{\prime}(x)\right\rangle$ is weakly upper semicontinuous for each $y \in H$;

(ii) for each $x \in H$, there exist a bounded subset $D_{x} \subset C$ and $z_{x} \in C$ such that for any $y \notin D_{x}$,

$$
\Theta\left(y, z_{x}\right)+\varphi\left(z_{x}\right)-\varphi(y)+\frac{1}{r}\left\langle K^{\prime}(y)-K^{\prime}(x), z_{x}-y\right\rangle<0 ;
$$

(iii) $0<\liminf _{n \rightarrow \infty} \beta_{n} \leq \limsup _{n \rightarrow \infty} \beta_{n}<1$;

(iv) $\left\{\lambda_{i, n}\right\} \subset\left[a_{i}, b_{i}\right] \subset\left(0,2 \eta_{i}\right)$ for $i=1,2$, and $\left\{r_{n}\right\} \subset[0,2 \zeta]$ satisfies

$$
0<\liminf _{n \rightarrow \infty} r_{n} \leq \limsup _{n \rightarrow \infty} r_{n}<2 \zeta
$$

Pick any $x_{0} \in H$ and set $C_{1}=C, x_{1}=P_{C_{1}} x_{0}$. Let $\left\{x_{n}\right\}$ be a sequence generated by the following algorithm:

$$
\left\{\begin{array}{l}
u_{n}=S_{r_{n}}^{(\Theta, \varphi)}\left(I-r_{n} A\right) x_{n}, \\
z_{n}=J_{R_{2}, \lambda_{2, n}}\left(I-\lambda_{2, n} B_{2}\right) J_{R_{1}, \lambda_{1, n}}\left(I-\lambda_{1, n} B_{1}\right) u_{n}, \\
k_{n}=\delta_{n} z_{n}+\left(1-\delta_{n}\right) S^{n} z_{n}, \\
y_{n}=\alpha_{n}\left(u+\gamma f\left(x_{n}\right)\right)+\beta_{n} k_{n}+\left(\left(1-\beta_{n}\right) I-\alpha_{n}(I+\mu V)\right) W_{n} z_{n}, \\
C_{n+1}=\left\{z \in C_{n}:\left\|y_{n}-z\right\|^{2} \leq\left\|x_{n}-z\right\|^{2}+\theta_{n}\right\}, \\
x_{n+1}=P_{C_{n+1}} x_{0}, \quad \forall n \geq 0,
\end{array}\right.
$$

where $\theta_{n}=\left(\alpha_{n}+\gamma_{n}\right) \Delta_{n} \varrho+c_{n} \varrho, \Delta_{n}=\sup \left\{\left\|x_{n}-p\right\|^{2}+\|u+(\gamma f-I-\mu V) p\|^{2}: p \in \Omega\right\}<\infty$, and $\varrho=\frac{1}{1-\sup _{n \geq 1} \alpha_{n}}<\infty$. If $S_{r}^{(\Theta, \varphi)}$ is firmly nonexpansive, then the following statements hold:

(I) $\left\{x_{n}\right\}$ converges strongly to $P_{\Omega} x_{0}$;

(II) $\left\{x_{n}\right\}$ converges strongly to $P_{\Omega} x_{0}$, which solves the optimization problem

$$
\min _{x \in \Omega} \frac{\mu}{2}\langle V x, x\rangle+\frac{1}{2}\|x-u\|^{2}-h(x)
$$

provided $\gamma_{n}+c_{n}+\left\|x_{n}-y_{n}\right\|=o\left(\alpha_{n}\right)$ additionally, where $h: H \rightarrow \mathbf{R}$ is the potential function of $\gamma f$.

Corollary 3.2 Let $C$ be a nonempty closed convex subset of a real Hilbert space H. Let $\Theta$ be a bifunction from $C \times C$ to $\mathbf{R}$ satisfying $(\mathrm{H} 1)-(\mathrm{H} 4)$ and $\varphi: C \rightarrow \mathbf{R}$ be a lower semicontinuous and convex functional. Let $R: C \rightarrow 2^{H}$ be a maximal monotone mapping and let $A: H \rightarrow H$ and $B: C \rightarrow H$ be $\zeta$-inverse strongly monotone and $\xi$-inverse-strongly monotone, respectively. Let $S: C \rightarrow C$ be a uniformly continuous asymptotically $\kappa$-strict pseudocontractive mapping in the intermediate sense for some $0 \leq \kappa<1$ with sequence $\left\{\gamma_{n}\right\} \subset[0, \infty)$ such that 
$\lim _{n \rightarrow \infty} \gamma_{n}=0$ and $\left\{c_{n}\right\} \subset[0, \infty)$ such that $\lim _{n \rightarrow \infty} c_{n}=0$. Let $\left\{T_{n}\right\}_{n=1}^{\infty}$ be a sequence of nonexpansive self-mappings on $C$ and $\left\{\lambda_{n}\right\}$ be a sequence in $(0, b]$ for some $b \in(0,1)$. Let $V$ be a $\bar{\gamma}$-strongly positive bounded linear operator and $f: H \rightarrow H$ be an l-Lipschitzian mapping with $\gamma l<(1+\mu) \bar{\gamma}$. Assume that $\Omega:=\left(\bigcap_{n=1}^{\infty} \operatorname{Fix}\left(T_{n}\right)\right) \cap \operatorname{GMEP}(\Theta, \varphi, A) \cap \mathrm{I}(B, R) \cap \operatorname{Fix}(S)$ is nonempty and bounded. Let $W_{n}$ be the $W$-mapping defined by (1.4) and $\left\{\alpha_{n}\right\},\left\{\beta_{n}\right\}$, and $\left\{\delta_{n}\right\}$ be three sequences in $(0,1)$ such that $\lim _{n \rightarrow \infty} \alpha_{n}=0$ and $\kappa \leq \delta_{n} \leq d<1$. Assume that:

(i) $K: H \rightarrow \mathbf{R}$ is strongly convex with constant $\sigma>0$ and its derivative $K^{\prime}$ is Lipschitz-continuous with constant $v>0$ such that the function $x \mapsto\left\langle y-x, K^{\prime}(x)\right\rangle$ is weakly upper semicontinuous for each $y \in H$;

(ii) for each $x \in H$, there exist a bounded subset $D_{x} \subset C$ and $z_{x} \in C$ such that for any $y \notin D_{x}$,

$$
\Theta\left(y, z_{x}\right)+\varphi\left(z_{x}\right)-\varphi(y)+\frac{1}{r}\left\langle K^{\prime}(y)-K^{\prime}(x), z_{x}-y\right\rangle<0
$$

(iii) $0<\liminf _{n \rightarrow \infty} \beta_{n} \leq \limsup _{n \rightarrow \infty} \beta_{n}<1$;

(iv) $\left\{\rho_{n}\right\} \subset[a, b] \subset(0,2 \xi)$, and $\left\{r_{n}\right\} \subset[0,2 \zeta]$ satisfies

$$
0<\liminf _{n \rightarrow \infty} r_{n} \leq \limsup _{n \rightarrow \infty} r_{n}<2 \zeta
$$

Pick any $x_{0} \in H$ and set $C_{1}=C, x_{1}=P_{C_{1}} x_{0}$. Let $\left\{x_{n}\right\}$ be a sequence generated by the following algorithm:

$$
\left\{\begin{array}{l}
u_{n}=S_{r_{n}}^{(\Theta, \varphi)}\left(I-r_{n} A\right) x_{n} \\
k_{n}=\delta_{n} J_{R, \rho_{n}}\left(I-\rho_{n} B\right) u_{n}+\left(1-\delta_{n}\right) S^{n} J_{R, \rho_{n}}\left(I-\rho_{n} B\right) u_{n} \\
y_{n}=\alpha_{n}\left(u+\gamma f\left(x_{n}\right)\right)+\beta_{n} k_{n}+\left(\left(1-\beta_{n}\right) I-\alpha_{n}(I+\mu V)\right) W_{n} J_{R, \rho_{n}}\left(I-\rho_{n} B\right) u_{n} \\
C_{n+1}=\left\{z \in C_{n}:\left\|y_{n}-z\right\|^{2} \leq\left\|x_{n}-z\right\|^{2}+\theta_{n}\right\} \\
x_{n+1}=P_{C_{n+1}} x_{0}, \quad \forall n \geq 0
\end{array}\right.
$$

where $\theta_{n}=\left(\alpha_{n}+\gamma_{n}\right) \Delta_{n} \varrho+c_{n} \varrho, \Delta_{n}=\sup \left\{\left\|x_{n}-p\right\|^{2}+\|u+(\gamma f-I-\mu V) p\|^{2}: p \in \Omega\right\}<\infty$, and $\varrho=\frac{1}{1-\sup _{n \geq 1} \alpha_{n}}<\infty$. If $S_{r}^{(\Theta, \varphi)}$ is firmly nonexpansive, then the following statements hold:

(I) $\left\{x_{n}\right\}$ converges strongly to $P_{\Omega} x_{0}$;

(II) $\left\{x_{n}\right\}$ converges strongly to $P_{\Omega} x_{0}$, which solves the optimization problem

$$
\min _{x \in \Omega} \frac{\mu}{2}\langle V x, x\rangle+\frac{1}{2}\|x-u\|^{2}-h(x)
$$

provided $\gamma_{n}+c_{n}+\left\|x_{n}-y_{n}\right\|=o\left(\alpha_{n}\right)$ additionally, where $h: H \rightarrow \mathbf{R}$ is the potential function of $\gamma f$.

\section{Weak convergence theorems}

In this section, we introduce and analyze another iterative algorithm for finding common solutions of a finite family of variational inclusions for maximal monotone and inversestrongly monotone mappings with the constraints of two problems: a generalized mixed equilibrium problem and a common fixed point problem of an infinite family of nonexpansive mappings and an asymptotically strict pseudocontractive mapping in the intermediate sense in a real Hilbert space. Under mild conditions imposed on the parameter sequences we will prove weak convergence of the proposed algorithm. 
Theorem 4.1 Let $C$ be a nonempty closed convex subset of a real Hilbert space H. Let $N$ be an integer. Let $\Theta$ be a bifunction from $C \times C$ to $\mathbf{R}$ satisfying $(\mathrm{H} 1)-(\mathrm{H} 4)$ and $\varphi: C \rightarrow \mathbf{R}$ be a lower semicontinuous and convex functional. Let $R_{i}: C \rightarrow 2^{H}$ be a maximal monotone mapping and let $A: H \rightarrow H$ and $B_{i}: C \rightarrow H$ be $\zeta$-inverse-strongly monotone and $\eta_{i}$-inverse-strongly monotone, respectively, where $i \in\{1,2, \ldots, N\}$. Let $S: C \rightarrow C$ be a uniformly continuous asymptotically $\kappa$-strict pseudocontractive mapping in the intermediate sense for some $0 \leq \kappa<1$ with sequences $\left\{\gamma_{n}\right\} \subset[0, \infty)$ and $\left\{c_{n}\right\} \subset[0, \infty)$. Let $\left\{T_{n}\right\}_{n=1}^{\infty}$ be a sequence of nonexpansive self-mappings on $C$ and $\left\{\lambda_{n}\right\}$ be a sequence in $(0, b]$ for some $b \in(0,1)$. Let $V$ be a $\bar{\gamma}$-strongly positive bounded linear operator and $f: H \rightarrow H$ be an l-Lipschitzian mapping with $\gamma l<(1+\mu) \bar{\gamma}$. Assume that $\Omega:=\left(\bigcap_{n=1}^{\infty} \operatorname{Fix}\left(T_{n}\right)\right) \cap$ $\operatorname{GMEP}(\Theta, \varphi, A) \cap\left(\bigcap_{i=1}^{N} \mathrm{I}\left(B_{i}, R_{i}\right)\right) \cap \operatorname{Fix}(S)$ is nonempty. Let $W_{n}$ be the $W$-mapping defined by (1.4) and $\left\{\alpha_{n}\right\},\left\{\beta_{n}\right\}$ and $\left\{\delta_{n}\right\}$ be three sequences in $(0,1)$ such that $0<\kappa+\varepsilon \leq \delta_{n} \leq d<1$. Assume that:

(i) $K: H \rightarrow \mathbf{R}$ is strongly convex with constant $\sigma>0$ and its derivative $K^{\prime}$ is Lipschitz-continuous with constant $v>0$ such that the function $x \mapsto\left\langle y-x, K^{\prime}(x)\right\rangle$ is weakly upper semicontinuous for each $y \in H$;

(ii) for each $x \in H$, there exist a bounded subset $D_{x} \subset C$ and $z_{x} \in C$ such that for any $y \notin D_{x}$,

$$
\Theta\left(y, z_{x}\right)+\varphi\left(z_{x}\right)-\varphi(y)+\frac{1}{r}\left\langle K^{\prime}(y)-K^{\prime}(x), z_{x}-y\right\rangle<0 ;
$$

(iii) $\sum_{n=1}^{\infty}\left(\alpha_{n}+\gamma_{n}+c_{n}\right)<\infty$ and $0<\liminf _{n \rightarrow \infty} \beta_{n} \leq \lim _{\lim } \sup _{n \rightarrow \infty} \beta_{n}<1$;

(iv) $\left\{\lambda_{i, n}\right\} \subset\left[a_{i}, b_{i}\right] \subset\left(0,2 \eta_{i}\right), \forall i \in\{1,2, \ldots, N\}$, and $\left\{r_{n}\right\} \subset[0,2 \zeta]$ satisfies

$$
0<\liminf _{n \rightarrow \infty} r_{n} \leq \limsup _{n \rightarrow \infty} r_{n}<2 \zeta
$$

Pick any $x_{1} \in H$ and let $\left\{x_{n}\right\}$ be a sequence generated by the following algorithm:

$$
\left\{\begin{array}{l}
u_{n}=S_{r_{n}}^{(\Theta, \varphi)}\left(I-r_{n} A\right) x_{n}, \\
z_{n}=J_{R_{N}, \lambda_{N, n}}\left(I-\lambda_{N, n} B_{N}\right) J_{R_{N-1}, \lambda_{N-1, n}}\left(I-\lambda_{N-1, n} B_{N-1}\right) \cdots J_{R_{1}, \lambda_{1, n}}\left(I-\lambda_{1, n} B_{1}\right) u_{n}, \\
k_{n}=\delta_{n} z_{n}+\left(1-\delta_{n}\right) S^{n} z_{n}, \\
x_{n+1}=\alpha_{n}\left(u+\gamma f\left(x_{n}\right)\right)+\beta_{n} k_{n}+\left(\left(1-\beta_{n}\right) I-\alpha_{n}(I+\mu V)\right) W_{n} z_{n}, \quad \forall n \geq 1 .
\end{array}\right.
$$

Then $\left\{x_{n}\right\}$ converges weakly to $w=\lim _{n \rightarrow \infty} P_{\Omega} x_{n}$ provided $S_{r}^{(\Theta, \varphi)}$ is firmly nonexpansive.

Proof First, let us show that $\lim _{n \rightarrow \infty}\left\|x_{n}-p\right\|$ exists for any $p \in \Omega$. Put

$$
\Lambda_{n}^{i}=J_{R_{i}, \lambda_{i, n}}\left(I-\lambda_{i, n} B_{i}\right) J_{R_{i-1}, \lambda_{i-1, n}}\left(I-\lambda_{i-1, n} B_{i-1}\right) \cdots J_{R_{1}, \lambda_{1, n}}\left(I-\lambda_{1, n} B_{1}\right)
$$

for all $i \in\{1,2, \ldots, N\}, n \geq 1$, and $\Lambda_{n}^{0}=I$, where $I$ is the identity mapping on $H$. Then we see that $z_{n}=\Lambda_{n}^{N} u_{n}$. Take $p \in \Omega$ arbitrarily. Similarly to the proof of Theorem 3.1, we obtain

$$
\begin{aligned}
& \left\|u_{n}-p\right\| \leq\left\|x_{n}-p\right\|, \\
& \left\|z_{n}-p\right\| \leq\left\|u_{n}-p\right\|, \\
& \left\|u_{n}-p\right\|^{2} \leq\left\|x_{n}-p\right\|^{2}+r_{n}\left(r_{n}-2 \zeta\right)\left\|A x_{n}-A p\right\|^{2},
\end{aligned}
$$




$$
\begin{gathered}
\left\|u_{n}-p\right\|^{2} \leq\left\|x_{n}-p\right\|^{2}-\left\|x_{n}-u_{n}\right\|^{2}+2 r_{n}\left\|A x_{n}-A p\right\|\left\|x_{n}-u_{n}\right\| \\
\left\|\Lambda_{n}^{i} u_{n}-p\right\|^{2} \leq\left\|x_{n}-p\right\|^{2}+\lambda_{i, n}\left(\lambda_{i, n}-2 \eta_{i}\right)\left\|B_{i} \Lambda_{n}^{i-1} u_{n}-B_{i} p\right\|^{2} \\
i \in\{1,2, \ldots, N\} \\
\left\|\Lambda_{n}^{i} u_{n}-p\right\|^{2} \leq\left\|x_{n}-p\right\|^{2}-\left\|\Lambda_{n}^{i-1} u_{n}-\Lambda_{n}^{i} u_{n}\right\|^{2} \\
+2 \lambda_{i, n}\left\|\Lambda_{n}^{i-1} u_{n}-\Lambda_{n}^{i} u_{n}\right\|\left\|B_{i} \Lambda_{n}^{i-1} u_{n}-B_{i} p\right\| \\
i \in\{1,2, \ldots, N\}
\end{gathered}
$$

By Lemma 2.2(b) we get

$$
\begin{aligned}
\| k_{n}- & p \|^{2} \\
= & \left\|\delta_{n}\left(z_{n}-p\right)+\left(1-\delta_{n}\right)\left(S^{n} z_{n}-p\right)\right\|^{2} \\
= & \delta_{n}\left\|z_{n}-p\right\|^{2}+\left(1-\delta_{n}\right)\left\|S^{n} z_{n}-p\right\|^{2}-\delta_{n}\left(1-\delta_{n}\right)\left\|z_{n}-S^{n} z_{n}\right\|^{2} \\
\leq & \delta_{n}\left\|z_{n}-p\right\|^{2}+\left(1-\delta_{n}\right)\left[\left(1+\gamma_{n}\right)\left\|z_{n}-p\right\|^{2}+\kappa\left\|z_{n}-S^{n} z_{n}\right\|^{2}+c_{n}\right] \\
& -\delta_{n}\left(1-\delta_{n}\right)\left\|z_{n}-S^{n} z_{n}\right\|^{2} \\
= & {\left[1+\gamma_{n}\left(1-\delta_{n}\right)\right]\left\|z_{n}-p\right\|^{2}+\left(1-\delta_{n}\right)\left(\kappa-\delta_{n}\right)\left\|z_{n}-S^{n} z_{n}\right\|^{2} } \\
& +\left(1-\delta_{n}\right) c_{n} \\
\leq & \left(1+\gamma_{n}\right)\left\|z_{n}-p\right\|^{2}+\left(1-\delta_{n}\right)\left(\kappa-\delta_{n}\right)\left\|z_{n}-S^{n} z_{n}\right\|^{2}+c_{n} \\
\leq & \left(1+\gamma_{n}\right)\left\|z_{n}-p\right\|^{2}+c_{n} .
\end{aligned}
$$

Repeating the same arguments as in the proof of Theorem 3.1 we have

$$
\left\|\left(1-\beta_{n}\right) I-\alpha_{n}(I+\mu V)\right\| \leq 1-\beta_{n}-\alpha_{n}-\alpha_{n} \mu \bar{\gamma}
$$

Then by Lemma 2.1 we deduce from (4.2), (4.3), (4.8), and $0 \leq \gamma l \leq(1+\mu) \bar{\gamma}$ that

$$
\begin{aligned}
\left\|x_{n+1}-p\right\|^{2} & \| \alpha_{n}(u+\gamma f(p)-\bar{V} p)+\alpha_{n} \gamma\left(f\left(x_{n}\right)-f(p)\right)+\beta_{n}\left(k_{n}-p\right) \\
& +\left(\left(1-\beta_{n}\right) I-\alpha_{n} \bar{V}\right)\left(W_{n} z_{n}-p\right) \|^{2} \\
\leq & \left\|\alpha_{n} \gamma\left(f\left(x_{n}\right)-f(p)\right)+\beta_{n}\left(k_{n}-p\right)+\left(\left(1-\beta_{n}\right) I-\alpha_{n} \bar{V}\right)\left(W_{n} z_{n}-p\right)\right\|^{2} \\
& +2 \alpha_{n}\left\langle(u+\gamma f(p)-\bar{V} p), x_{n+1}-p\right\rangle \\
\leq & {\left[\alpha_{n} \gamma\left\|f\left(x_{n}\right)-f(p)\right\|+\beta_{n}\left\|k_{n}-p\right\|+\left\|\left(\left(1-\beta_{n}\right) I-\alpha_{n} \bar{V}\right)\left(W_{n} z_{n}-p\right)\right\|\right]^{2} } \\
& +2 \alpha_{n}\|u+\gamma f(p)-\bar{V} p\|\left\|x_{n+1}-p\right\| \\
\leq & {\left[\alpha_{n} \gamma l\left\|x_{n}-p\right\|+\beta_{n}\left\|k_{n}-p\right\|+\left(1-\beta_{n}-\alpha_{n}-\alpha_{n} \mu \bar{\gamma}\right)\left\|W_{n} z_{n}-p\right\|\right]^{2} } \\
& +2 \alpha_{n}\|u+\gamma f(p)-\bar{V} p\|\left\|x_{n+1}-p\right\| \\
\leq & {\left[\alpha_{n}(1+\mu) \bar{\gamma}\left\|x_{n}-p\right\|+\beta_{n}\left\|k_{n}-p\right\|+\left(1-\beta_{n}-\alpha_{n}(1+\mu) \bar{\gamma}\right)\left\|z_{n}-p\right\|\right]^{2} }
\end{aligned}
$$




$$
\begin{aligned}
& +2 \alpha_{n}\|u+\gamma f(p)-\bar{V} p\|\left\|x_{n+1}-p\right\| \\
\leq & \alpha_{n}(1+\mu) \bar{\gamma}\left\|x_{n}-p\right\|^{2}+\beta_{n}\left\|k_{n}-p\right\|^{2}+\left(1-\beta_{n}-\alpha_{n}(1+\mu) \bar{\gamma}\right)\left\|z_{n}-p\right\|^{2} \\
& +2 \alpha_{n}\|u+\gamma f(p)-\bar{V} p\|\left\|x_{n+1}-p\right\| \\
\leq & \alpha_{n}(1+\mu) \bar{\gamma}\left\|x_{n}-p\right\|^{2}+\beta_{n}\left(\left(1+\gamma_{n}\right)\left\|z_{n}-p\right\|^{2}+c_{n}\right) \\
& +\left(1-\beta_{n}-\alpha_{n}(1+\mu) \bar{\gamma}\right)\left\|z_{n}-p\right\|^{2}+2 \alpha_{n}\|u+\gamma f(p)-\bar{V} p\|\left\|x_{n+1}-p\right\| \\
\leq & \alpha_{n}(1+\mu) \bar{\gamma}\left\|x_{n}-p\right\|^{2}+\beta_{n}\left(\left(1+\gamma_{n}\right)\left\|x_{n}-p\right\|^{2}+c_{n}\right) \\
& +\left(1-\beta_{n}-\alpha_{n}(1+\mu) \bar{\gamma}\right)\left\|x_{n}-p\right\|^{2}+2 \alpha_{n}\|u+\gamma f(p)-\bar{V} p\|\left\|x_{n+1}-p\right\| \\
\leq & \alpha_{n}(1+\mu) \bar{\gamma}\left(\left(1+\gamma_{n}\right)\left\|x_{n}-p\right\|^{2}+c_{n}\right)+\beta_{n}\left(\left(1+\gamma_{n}\right)\left\|x_{n}-p\right\|^{2}+c_{n}\right) \\
& +\left(1-\beta_{n}-\alpha_{n}(1+\mu) \bar{\gamma}\right)\left(\left(1+\gamma_{n}\right)\left\|x_{n}-p\right\|^{2}+c_{n}\right) \\
& +2 \alpha_{n}\|u+\gamma f(p)-\bar{V} p\|\left\|x_{n+1}-p\right\| \\
= & \left(1+\gamma_{n}\right)\left\|x_{n}-p\right\|^{2}+c_{n}+2 \alpha_{n}\|u+\gamma f(p)-\bar{V} p\|\left\|x_{n+1}-p\right\| \\
\leq & \left(1+\gamma_{n}\right)\left\|x_{n}-p\right\|^{2}+c_{n}+\alpha_{n}\left(\|u+\gamma f(p)-\bar{V} p\|^{2}+\left\|x_{n+1}-p\right\|^{2}\right),
\end{aligned}
$$

which hence yields

$$
\begin{aligned}
& \left\|x_{n+1}-p\right\|^{2} \\
& \quad \leq \frac{1+\gamma_{n}}{1-\alpha_{n}}\left\|x_{n}-p\right\|^{2}+\frac{\alpha_{n}}{1-\alpha_{n}}\|u+\gamma f(p)-\bar{V} p\|^{2}+\frac{1}{1-\alpha_{n}} c_{n} \\
& \quad=\left(1+\frac{\alpha_{n}+\gamma_{n}}{1-\alpha_{n}}\right)\left\|x_{n}-p\right\|^{2}+\frac{\alpha_{n}}{1-\alpha_{n}}\|u+\gamma f(p)-\bar{V} p\|^{2}+\frac{1}{1-\alpha_{n}} c_{n} \\
& \quad \leq\left[1+\left(\alpha_{n}+\gamma_{n}\right) \varrho\right]\left\|x_{n}-p\right\|^{2}+\alpha_{n} \varrho\|u+\gamma f(p)-\bar{V} p\|^{2}+\varrho c_{n},
\end{aligned}
$$

where $\varrho=\frac{1}{1-\sup _{n \geq 1} \alpha_{n}}<\infty$ (due to $\left\{\alpha_{n}\right\} \subset(0,1)$ and $\lim _{n \rightarrow \infty} \alpha_{n}=0$ ). By Lemma 2.8, we see from $\sum_{n=1}^{\infty}\left(\alpha_{n}+\gamma_{n}+c_{n}\right)<\infty$ that $\lim _{n \rightarrow \infty}\left\|x_{n}-p\right\|$ exists. Thus $\left\{x_{n}\right\}$ is bounded and so are the sequences $\left\{u_{n}\right\},\left\{z_{n}\right\}$, and $\left\{k_{n}\right\}$.

Also, utilizing Lemmas 2.1 and 2.2(b) we obtain from (4.2), (4.3), and (4.8)

$$
\begin{aligned}
&\left\|x_{n+1}-p\right\|^{2} \\
&=\left\|\alpha_{n}\left(u+\gamma f\left(x_{n}\right)-\bar{V} W_{n} z_{n}\right)+\beta_{n}\left(k_{n}-p\right)+\left(1-\beta_{n}\right)\left(W_{n} z_{n}-p\right)\right\|^{2} \\
& \leq\left\|\beta_{n}\left(k_{n}-p\right)+\left(1-\beta_{n}\right)\left(W_{n} z_{n}-p\right)\right\|^{2} \\
&+2 \alpha_{n}\left\langle u+\gamma f\left(x_{n}\right)-\bar{V} W_{n} z_{n}, x_{n+1}-p\right\rangle \\
&= \beta_{n}\left\|k_{n}-p\right\|^{2}+\left(1-\beta_{n}\right)\left\|W_{n} z_{n}-p\right\|^{2}-\beta_{n}\left(1-\beta_{n}\right)\left\|k_{n}-W_{n} z_{n}\right\|^{2} \\
&+2 \alpha_{n}\left\|u+\gamma f\left(x_{n}\right)-\bar{V} W_{n} z_{n}\right\|\left\|x_{n+1}-p\right\| \\
& \leq \beta_{n}\left\|k_{n}-p\right\|^{2}+\left(1-\beta_{n}\right)\left\|z_{n}-p\right\|^{2}-\beta_{n}\left(1-\beta_{n}\right)\left\|k_{n}-W_{n} z_{n}\right\|^{2} \\
&+2 \alpha_{n}\left\|u+\gamma f\left(x_{n}\right)-\bar{V} W_{n} z_{n}\right\|\left\|x_{n+1}-p\right\| \\
& \leq \beta_{n}\left(\left(1+\gamma_{n}\right)\left\|z_{n}-p\right\|^{2}+c_{n}\right)+\left(1-\beta_{n}\right)\left\|z_{n}-p\right\|^{2} \\
&-\beta_{n}\left(1-\beta_{n}\right)\left\|k_{n}-W_{n} z_{n}\right\|^{2}
\end{aligned}
$$




$$
\begin{aligned}
& +2 \alpha_{n}\left\|u+\gamma f\left(x_{n}\right)-\bar{V} W_{n} z_{n}\right\|\left\|x_{n+1}-p\right\| \\
\leq & \beta_{n}\left(\left(1+\gamma_{n}\right)\left\|z_{n}-p\right\|^{2}+c_{n}\right)+\left(1-\beta_{n}\right)\left(\left(1+\gamma_{n}\right)\left\|z_{n}-p\right\|^{2}+c_{n}\right) \\
& -\beta_{n}\left(1-\beta_{n}\right)\left\|k_{n}-W_{n} z_{n}\right\|^{2} \\
& +2 \alpha_{n}\left\|u+\gamma f\left(x_{n}\right)-\bar{V} W_{n} z_{n}\right\|\left\|x_{n+1}-p\right\| \\
\leq & \left(1+\gamma_{n}\right)\left\|z_{n}-p\right\|^{2}+c_{n}-\beta_{n}\left(1-\beta_{n}\right)\left\|k_{n}-W_{n} z_{n}\right\|^{2} \\
& +2 \alpha_{n}\left\|u+\gamma f\left(x_{n}\right)-\bar{V} W_{n} z_{n}\right\|\left\|x_{n+1}-p\right\| \\
\leq & \left(1+\gamma_{n}\right)\left\|x_{n}-p\right\|^{2}+c_{n}-\beta_{n}\left(1-\beta_{n}\right)\left\|k_{n}-W_{n} z_{n}\right\|^{2} \\
& +2 \alpha_{n}\left\|u+\gamma f\left(x_{n}\right)-\bar{V} W_{n} z_{n}\right\|\left\|x_{n+1}-p\right\|,
\end{aligned}
$$

which leads to

$$
\begin{aligned}
\beta_{n}\left(1-\beta_{n}\right)\left\|k_{n}-W_{n} z_{n}\right\|^{2} \\
\leq \\
\quad\left\|x_{n}-p\right\|^{2}-\left\|x_{n+1}-p\right\|^{2}+\gamma_{n}\left\|x_{n}-p\right\|^{2}+c_{n} \\
\quad+2 \alpha_{n}\left\|u+\gamma f\left(x_{n}\right)-\bar{V} W_{n} z_{n}\right\|\left\|x_{n+1}-p\right\| .
\end{aligned}
$$

Since $\lim _{n \rightarrow \infty} \alpha_{n}=0, \lim _{n \rightarrow \infty} \gamma_{n}=0$, and $\lim _{n \rightarrow \infty} c_{n}=0$, it follows from the existence of $\lim _{n \rightarrow \infty}\left\|x_{n}-p\right\|$ and condition (iii) that

$$
\lim _{n \rightarrow \infty}\left\|k_{n}-W_{n} z_{n}\right\|=0
$$

Note that

$$
x_{n+1}-k_{n}=\alpha_{n}\left(u+\gamma f\left(x_{n}\right)-\bar{V} W_{n} z_{n}\right)+\left(1-\beta_{n}\right)\left(W_{n} z_{n}-k_{n}\right)
$$

which yields

$$
\begin{aligned}
\left\|x_{n+1}-k_{n}\right\| & \leq \alpha_{n}\left\|u+\gamma f\left(x_{n}\right)-\bar{V} W_{n} z_{n}\right\|+\left(1-\beta_{n}\right)\left\|W_{n} z_{n}-k_{n}\right\| \\
& \leq \alpha_{n}\left\|u+\gamma f\left(x_{n}\right)-\bar{V} W_{n} z_{n}\right\|+\left\|W_{n} z_{n}-k_{n}\right\| .
\end{aligned}
$$

So, from (4.11) and $\lim _{n \rightarrow \infty} \alpha_{n}=0$, we get

$$
\lim _{n \rightarrow \infty}\left\|x_{n+1}-k_{n}\right\|=0
$$

In the meantime, we conclude from (4.2), (4.3), (4.8), and (4.10) that

$$
\begin{aligned}
&\left\|x_{n+1}-p\right\|^{2} \\
& \leq \beta_{n}\left\|k_{n}-p\right\|^{2}+\left(1-\beta_{n}\right)\left\|z_{n}-p\right\|^{2}-\beta_{n}\left(1-\beta_{n}\right)\left\|k_{n}-W_{n} z_{n}\right\|^{2} \\
&+2 \alpha_{n}\left\|u+\gamma f\left(x_{n}\right)-\bar{V} W_{n} z_{n}\right\|\left\|x_{n+1}-p\right\| \\
& \leq \beta_{n}\left\|k_{n}-p\right\|^{2}+\left(1-\beta_{n}\right)\left\|z_{n}-p\right\|^{2}+2 \alpha_{n}\left\|u+\gamma f\left(x_{n}\right)-\bar{V} W_{n} z_{n}\right\|\left\|x_{n+1}-p\right\| \\
& \leq \beta_{n}\left[\left(1+\gamma_{n}\right)\left\|z_{n}-p\right\|^{2}+\left(1-\delta_{n}\right)\left(\kappa-\delta_{n}\right)\left\|z_{n}-S^{n} z_{n}\right\|^{2}+c_{n}\right]
\end{aligned}
$$




$$
\begin{aligned}
& +\left(1-\beta_{n}\right)\left\|z_{n}-p\right\|^{2}+2 \alpha_{n}\left\|u+\gamma f\left(x_{n}\right)-\bar{V} W_{n} z_{n}\right\|\left\|x_{n+1}-p\right\| \\
\leq & \beta_{n}\left[\left(1+\gamma_{n}\right)\left\|z_{n}-p\right\|^{2}+\left(1-\delta_{n}\right)\left(\kappa-\delta_{n}\right)\left\|z_{n}-S^{n} z_{n}\right\|^{2}+c_{n}\right] \\
& +\left(1-\beta_{n}\right)\left(\left(1+\gamma_{n}\right)\left\|z_{n}-p\right\|^{2}+c_{n}\right) \\
& +2 \alpha_{n}\left\|u+\gamma f\left(x_{n}\right)-\bar{V} W_{n} z_{n}\right\|\left\|x_{n+1}-p\right\| \\
\leq & \left(1+\gamma_{n}\right)\left\|z_{n}-p\right\|^{2}+\beta_{n}\left(1-\delta_{n}\right)\left(\kappa-\delta_{n}\right)\left\|z_{n}-S^{n} z_{n}\right\|^{2}+c_{n} \\
& +2 \alpha_{n}\left\|u+\gamma f\left(x_{n}\right)-\bar{V} W_{n} z_{n}\right\|\left\|x_{n+1}-p\right\| \\
\leq & \left(1+\gamma_{n}\right)\left\|x_{n}-p\right\|^{2}+\beta_{n}\left(1-\delta_{n}\right)\left(\kappa-\delta_{n}\right)\left\|z_{n}-S^{n} z_{n}\right\|^{2}+c_{n} \\
& +2 \alpha_{n}\left\|u+\gamma f\left(x_{n}\right)-\bar{V} W_{n} z_{n}\right\|\left\|x_{n+1}-p\right\|,
\end{aligned}
$$

which, together with $0<\kappa+\epsilon \leq \delta_{n} \leq d<1$, implies that

$$
\begin{aligned}
(1-d) \epsilon \beta_{n}\left\|z_{n}-S^{n} z_{n}\right\|^{2} \leq & \beta_{n}\left(1-\delta_{n}\right)\left(\delta_{n}-\kappa\right)\left\|z_{n}-S^{n} z_{n}\right\|^{2} \\
\leq & \left\|x_{n}-p\right\|^{2}-\left\|x_{n+1}-p\right\|^{2}+\gamma_{n}\left\|x_{n}-p\right\|^{2}+c_{n} \\
& +2 \alpha_{n}\left\|u+\gamma f\left(x_{n}\right)-\bar{V} W_{n} z_{n}\right\|\left\|x_{n+1}-p\right\| .
\end{aligned}
$$

Consequently, from $\lim _{n \rightarrow \infty} \alpha_{n}=0, \lim _{n \rightarrow \infty} \gamma_{n}=0, \lim _{n \rightarrow \infty} c_{n}=0$, condition (iii), and the existence of $\lim _{n \rightarrow \infty}\left\|x_{n}-p\right\|$, we get

$$
\lim _{n \rightarrow \infty}\left\|z_{n}-S^{n} z_{n}\right\|=0
$$

Since $k_{n}-z_{n}=\left(1-\delta_{n}\right)\left(S^{n} z_{n}-z_{n}\right)$, from (4.13) we have

$$
\lim _{n \rightarrow \infty}\left\|k_{n}-z_{n}\right\|=0 .
$$

Combining (4.4), (4.8), and (4.10), we have

$$
\begin{aligned}
\left\|x_{n+1}-p\right\|^{2} \leq & \beta_{n}\left(\left(1+\gamma_{n}\right)\left\|z_{n}-p\right\|^{2}+c_{n}\right)+\left(1-\beta_{n}\right)\left\|z_{n}-p\right\|^{2}-\beta_{n}\left(1-\beta_{n}\right)\left\|k_{n}-W_{n} z_{n}\right\|^{2} \\
& +2 \alpha_{n}\left\|u+\gamma f\left(x_{n}\right)-\bar{V} W_{n} z_{n}\right\|\left\|x_{n+1}-p\right\| \\
\leq & \beta_{n}\left(\left(1+\gamma_{n}\right)\left\|z_{n}-p\right\|^{2}+c_{n}\right)+\left(1-\beta_{n}\right)\left(\left(1+\gamma_{n}\right)\left\|z_{n}-p\right\|^{2}+c_{n}\right) \\
& +2 \alpha_{n}\left\|u+\gamma f\left(x_{n}\right)-\bar{V} W_{n} z_{n}\right\|\left\|x_{n+1}-p\right\| \\
= & \left(1+\gamma_{n}\right)\left\|z_{n}-p\right\|^{2}+c_{n}+2 \alpha_{n}\left\|u+\gamma f\left(x_{n}\right)-\bar{V} W_{n} z_{n}\right\|\left\|x_{n+1}-p\right\| \\
\leq & \left\|u_{n}-p\right\|^{2}+\gamma_{n}\left\|x_{n}-p\right\|^{2}+c_{n}+2 \alpha_{n}\left\|u+\gamma f\left(x_{n}\right)-\bar{V} W_{n} z_{n}\right\|\left\|x_{n+1}-p\right\| \\
\leq & \left\|x_{n}-p\right\|^{2}+r_{n}\left(r_{n}-2 \zeta\right)\left\|A x_{n}-A p\right\|^{2}+\gamma_{n}\left\|x_{n}-p\right\|^{2}+c_{n} \\
& +2 \alpha_{n}\left\|u+\gamma f\left(x_{n}\right)-\bar{V} W_{n} z_{n}\right\|\left\|x_{n+1}-p\right\|
\end{aligned}
$$

which implies

$$
\begin{aligned}
r_{n}\left(2 \zeta-r_{n}\right)\left\|A x_{n}-A p\right\|^{2} \\
\leq \\
\quad\left\|x_{n}-p\right\|^{2}-\left\|x_{n+1}-p\right\|^{2}+\gamma_{n}\left\|x_{n}-p\right\|^{2}+c_{n} \\
\quad+2 \alpha_{n}\left\|u+\gamma f\left(x_{n}\right)-\bar{V} W_{n} z_{n}\right\|\left\|x_{n+1}-p\right\| .
\end{aligned}
$$


From condition (iv), $\lim _{n \rightarrow \infty} \alpha_{n}=0, \lim _{n \rightarrow \infty} \gamma_{n}=0, \lim _{n \rightarrow \infty} c_{n}=0$, and the existence of $\lim _{n \rightarrow \infty}\left\|x_{n}-p\right\|$, we get

$$
\lim _{n \rightarrow \infty}\left\|A x_{n}-A p\right\|=0 .
$$

Combining (4.5), (4.8), and (4.10), we have

$$
\begin{aligned}
\left\|x_{n+1}-p\right\|^{2} \leq & \left\|z_{n}-p\right\|^{2}+\gamma_{n}\left\|z_{n}-p\right\|^{2}+c_{n} \\
& +2 \alpha_{n}\left\|u+\gamma f\left(x_{n}\right)-\bar{V} W_{n} z_{n}\right\|\left\|x_{n+1}-p\right\| \\
\leq & \left\|u_{n}-p\right\|^{2}+\gamma_{n}\left\|x_{n}-p\right\|^{2}+c_{n} \\
& +2 \alpha_{n}\left\|u+\gamma f\left(x_{n}\right)-\bar{V} W_{n} z_{n}\right\|\left\|x_{n+1}-p\right\| \\
\leq & \left\|x_{n}-p\right\|^{2}-\left\|x_{n}-u_{n}\right\|^{2}+2 r_{n}\left\|A x_{n}-A p\right\|\left\|x_{n}-u_{n}\right\| \\
& +\gamma_{n}\left\|x_{n}-p\right\|^{2}+c_{n}+2 \alpha_{n}\left\|u+\gamma f\left(x_{n}\right)-\bar{V} W_{n} z_{n}\right\|\left\|x_{n+1}-p\right\|,
\end{aligned}
$$

which implies

$$
\begin{aligned}
\left\|x_{n}-u_{n}\right\|^{2} \leq & \left\|x_{n}-p\right\|^{2}-\left\|x_{n+1}-p\right\|^{2}+2 r_{n}\left\|A x_{n}-A p\right\|\left\|x_{n}-u_{n}\right\| \\
& +\gamma_{n}\left\|x_{n}-p\right\|^{2}+c_{n}+2 \alpha_{n}\left\|u+\gamma f\left(x_{n}\right)-\bar{V} W_{n} z_{n}\right\|\left\|x_{n+1}-p\right\| .
\end{aligned}
$$

From (4.15), $\lim _{n \rightarrow \infty} \alpha_{n}=0, \lim _{n \rightarrow \infty} \gamma_{n}=0, \lim _{n \rightarrow \infty} c_{n}=0$, and the existence of $\lim _{n \rightarrow \infty}\left\|x_{n}-p\right\|$, we obtain

$$
\lim _{n \rightarrow \infty}\left\|x_{n}-u_{n}\right\|=0
$$

Combining (4.6), (4.8), and (4.10), we have

$$
\begin{aligned}
&\left\|x_{n+1}-p\right\|^{2} \\
& \leq\left\|z_{n}-p\right\|^{2}+\gamma_{n}\left\|z_{n}-p\right\|^{2}+c_{n}+2 \alpha_{n}\left\|u+\gamma f\left(x_{n}\right)-\bar{V} W_{n} z_{n}\right\|\left\|x_{n+1}-p\right\| \\
& \leq\left\|\Lambda_{n}^{i} u_{n}-p\right\|^{2}+\gamma_{n}\left\|z_{n}-p\right\|^{2}+c_{n}+2 \alpha_{n}\left\|u+\gamma f\left(x_{n}\right)-\bar{V} W_{n} z_{n}\right\|\left\|x_{n+1}-p\right\| \\
& \leq\left\|x_{n}-p\right\|^{2}+\lambda_{i, n}\left(\lambda_{i, n}-2 \eta_{i}\right)\left\|B_{i} \Lambda_{n}^{i-1} u_{n}-B_{i} p\right\|^{2}+\gamma_{n}\left\|x_{n}-p\right\|^{2}+c_{n} \\
&+2 \alpha_{n}\left\|u+\gamma f\left(x_{n}\right)-\bar{V} W_{n} z_{n}\right\|\left\|x_{n+1}-p\right\|,
\end{aligned}
$$

which implies

$$
\begin{aligned}
& \lambda_{i, n}\left(\lambda_{i, n}-2 \eta_{i}\right)\left\|B_{i} \Lambda_{n}^{i-1} u_{n}-B_{i} p\right\|^{2} \\
& \leq\left\|x_{n}-p\right\|^{2}-\left\|x_{n+1}-p\right\|^{2}+\gamma_{n}\left\|x_{n}-p\right\|^{2}+c_{n} \\
&+2 \alpha_{n}\left\|u+\gamma f\left(x_{n}\right)-\bar{V} W_{n} z_{n}\right\|\left\|x_{n+1}-p\right\| .
\end{aligned}
$$

From $\left\{\lambda_{i, n}\right\} \subset\left[a_{i}, b_{i}\right] \subset\left(0,2 \eta_{i}\right), i \in\{1,2, \ldots, N\}, \lim _{n \rightarrow \infty} \alpha_{n}=0, \lim _{n \rightarrow \infty} \gamma_{n}=0, \lim _{n \rightarrow \infty} c_{n}=$ 0 , and the existence of $\lim _{n \rightarrow \infty}\left\|x_{n}-p\right\|$, we obtain

$$
\lim _{n \rightarrow \infty}\left\|B_{i} \Lambda_{n}^{i-1} u_{n}-B_{i} p\right\|=0, \quad i \in\{1,2, \ldots, N\}
$$


Combining (4.7), (4.8), and (4.10), we get

$$
\begin{aligned}
& \left\|x_{n+1}-p\right\|^{2} \\
& \leq\left\|z_{n}-p\right\|^{2}+\gamma_{n}\left\|z_{n}-p\right\|^{2}+c_{n}+2 \alpha_{n}\left\|u+\gamma f\left(x_{n}\right)-\bar{V} W_{n} z_{n}\right\|\left\|x_{n+1}-p\right\| \\
& \leq\left\|\Lambda_{n}^{i} u_{n}-p\right\|^{2}+\gamma_{n}\left\|x_{n}-p\right\|^{2}+c_{n}+2 \alpha_{n}\left\|u+\gamma f\left(x_{n}\right)-\bar{V} W_{n} z_{n}\right\|\left\|x_{n+1}-p\right\| \\
& \leq\left\|x_{n}-p\right\|^{2}-\left\|\Lambda_{n}^{i-1} u_{n}-\Lambda_{n}^{i} u_{n}\right\|^{2}+2 \lambda_{i, n}\left\|\Lambda_{n}^{i-1} u_{n}-\Lambda_{n}^{i} u_{n}\right\|\left\|B_{i} \Lambda_{n}^{i-1} u_{n}-B_{i} p\right\| \\
& \quad+\gamma_{n}\left\|x_{n}-p\right\|^{2}+c_{n}+2 \alpha_{n}\left\|u+\gamma f\left(x_{n}\right)-\bar{V} W_{n} z_{n}\right\|\left\|x_{n+1}-p\right\|,
\end{aligned}
$$

which implies

$$
\begin{aligned}
& \left\|\Lambda_{n}^{i-1} u_{n}-\Lambda_{n}^{i} u_{n}\right\|^{2} \\
& \quad \leq\left\|x_{n}-p\right\|^{2}-\left\|x_{n+1}-p\right\|^{2}+2 \lambda_{i, n}\left\|\Lambda_{n}^{i-1} u_{n}-\Lambda_{n}^{i} u_{n}\right\|\left\|B_{i} \Lambda_{n}^{i-1} u_{n}-B_{i} p\right\| \\
& \quad+\gamma_{n}\left\|x_{n}-p\right\|^{2}+c_{n}+2 \alpha_{n}\left\|u+\gamma f\left(x_{n}\right)-\bar{V} W_{n} z_{n}\right\|\left\|x_{n+1}-p\right\| .
\end{aligned}
$$

From (4.17), $\lim _{n \rightarrow \infty} \alpha_{n}=0, \lim _{n \rightarrow \infty} \gamma_{n}=0, \lim _{n \rightarrow \infty} c_{n}=0$, and the existence of $\lim _{n \rightarrow \infty}\left\|x_{n}-p\right\|$, we obtain

$$
\lim _{n \rightarrow \infty}\left\|\Lambda_{n}^{i-1} u_{n}-\Lambda_{n}^{i} u_{n}\right\|=0, \quad i \in\{1,2, \ldots, N\}
$$

By (4.18), we have

$$
\begin{aligned}
\left\|u_{n}-z_{n}\right\| & =\left\|\Lambda_{n}^{0} u_{n}-\Lambda_{n}^{N} u_{n}\right\| \\
& \leq\left\|\Lambda_{n}^{0} u_{n}-\Lambda_{n}^{1} u_{n}\right\|+\left\|\Lambda_{n}^{1} u_{n}-\Lambda_{n}^{2} u_{n}\right\|+\cdots+\left\|\Lambda_{n}^{N-1} u_{n}-\Lambda_{n}^{N} u_{n}\right\| \\
& \rightarrow 0 \quad \text { as } n \rightarrow \infty
\end{aligned}
$$

From (4.16) and (4.19), we have

$$
\begin{aligned}
\left\|x_{n}-z_{n}\right\| & \leq\left\|x_{n}-u_{n}\right\|+\left\|u_{n}-z_{n}\right\| \\
& \rightarrow 0 \quad \text { as } n \rightarrow \infty
\end{aligned}
$$

By (4.14) and (4.20), we obtain

$$
\begin{aligned}
\left\|k_{n}-x_{n}\right\| & \leq\left\|k_{n}-z_{n}\right\|+\left\|z_{n}-x_{n}\right\| \\
& \rightarrow 0 \quad \text { as } n \rightarrow \infty
\end{aligned}
$$

which, together with (4.12) and (4.21), implies that

$$
\begin{aligned}
\left\|x_{n+1}-x_{n}\right\| & \leq\left\|x_{n+1}-k_{n}\right\|+\left\|k_{n}-x_{n}\right\| \\
& \rightarrow 0 \quad \text { as } n \rightarrow \infty .
\end{aligned}
$$

On the other hand, we observe that

$$
\left\|z_{n+1}-z_{n}\right\| \leq\left\|z_{n+1}-x_{n+1}\right\|+\left\|x_{n+1}-x_{n}\right\|+\left\|x_{n}-z_{n}\right\| .
$$


By (4.20) and (4.22), we have

$$
\lim _{n \rightarrow \infty}\left\|z_{n+1}-z_{n}\right\|=0
$$

We note that

$$
\begin{aligned}
\left\|z_{n}-S z_{n}\right\| \leq & \left\|z_{n}-z_{n+1}\right\|+\left\|z_{n+1}-S^{n+1} z_{n+1}\right\|+\left\|S^{n+1} z_{n+1}-S^{n+1} z_{n}\right\| \\
& +\left\|S^{n+1} z_{n}-S z_{n}\right\| .
\end{aligned}
$$

From (4.13), (4.23), Lemma 2.4, and the uniform continuity of $S$, we obtain

$$
\lim _{n \rightarrow \infty}\left\|z_{n}-S z_{n}\right\|=0
$$

In addition, note that

$$
\left\|z_{n}-W z_{n}\right\| \leq\left\|z_{n}-k_{n}\right\|+\left\|k_{n}-W_{n} z_{n}\right\|+\left\|W_{n} z_{n}-W z_{n}\right\|
$$

So, from (4.11), (4.14), and Remark 2.3 it follows that

$$
\lim _{n \rightarrow \infty}\left\|z_{n}-W z_{n}\right\|=0
$$

Since $\left\{x_{n}\right\}$ is bounded, there exists a subsequence $\left\{x_{n_{i}}\right\}$ of $\left\{x_{n}\right\}$ which converges weakly to $w$. From (4.20) and (4.21), we have $z_{n_{i}} \rightarrow w$ and $k_{n_{i}} \rightarrow w$. From (4.24) and the uniform continuity of $S$, we have $\lim _{n \rightarrow \infty}\left\|z_{n}-S^{m} z_{n}\right\|=0$ for any $m \geq 1$. So, from Lemma 2.6, we have $w \in \operatorname{Fix}(S)$. In the meantime, by (4.25) and Lemma 2.13, we get $w \in \operatorname{Fix}(W)=\bigcap_{n=1}^{\infty} \operatorname{Fix}\left(T_{n}\right)$ (due to Lemma 2.12). Utilizing similar arguments to those in the proof of Theorem 3.1, we can derive $w \in \operatorname{GMEP}(\Theta, \varphi, A) \cap\left(\bigcap_{i=1}^{N} \mathrm{I}\left(B_{i}, R_{i}\right)\right)$. Consequently, $w \in \Omega$. This shows that $\omega_{w}\left(x_{n}\right) \subset \Omega$.

Next let us show that $\omega_{w}\left(x_{n}\right)$ is a single-point set. As a matter of fact, let $\left\{x_{n_{j}}\right\}$ be another subsequence of $\left\{x_{n}\right\}$ such that $x_{n_{j}} \rightarrow w^{\prime}$. Then we get $w^{\prime} \in \Omega$. If $w \neq w^{\prime}$, from the Opial condition, we have

$$
\begin{aligned}
\lim _{n \rightarrow \infty}\left\|x_{n}-w\right\| & =\lim _{i \rightarrow \infty}\left\|x_{n_{i}}-w\right\| \\
& <\lim _{i \rightarrow \infty}\left\|x_{n_{i}}-w^{\prime}\right\| \\
& =\lim _{n \rightarrow \infty}\left\|x_{n}-w^{\prime}\right\| \\
& =\lim _{j \rightarrow \infty}\left\|x_{n_{j}}-w^{\prime}\right\| \\
& <\lim _{j \rightarrow \infty}\left\|x_{n_{j}}-w\right\| \\
& =\lim _{n \rightarrow \infty}\left\|x_{n}-w\right\| .
\end{aligned}
$$

This attains a contradiction. So we have $w=w^{\prime}$. Put $v_{n}=P_{\Omega} x_{n}$. Since $w \in \Omega$, we have $\left\langle x_{n}-v_{n}, v_{n}-w\right\rangle \geq 0$. By Lemma 2.9, we see that $\left\{v_{n}\right\}$ converges strongly to some $w_{0} \in \Omega$. 
Since $\left\{x_{n}\right\}$ converges weakly to $w$, we have

$$
\left\langle w-w_{0}, w_{0}-w\right\rangle \geq 0 .
$$

Therefore we obtain $w=w_{0}=\lim _{n \rightarrow \infty} P_{\Omega} x_{n}$. This completes the proof.

Corollary 4.1 Let $C$ be a nonempty closed convex subset of a real Hilbert space H. Let $\Theta$ be a bifunction from $C \times C$ to $\mathbf{R}$ satisfying $(\mathrm{H} 1)-(\mathrm{H} 4)$ and $\varphi: C \rightarrow \mathbf{R}$ be a lower semicontinuous and convex functional. Let $R_{i}: C \rightarrow 2^{H}$ be a maximal monotone mapping and let $A: H \rightarrow H$ and $B_{i}: C \rightarrow H$ be $\zeta$-inverse-strongly monotone and $\eta_{i}$-inverse-strongly monotone, respectively, for $i=1,2$. Let $S: C \rightarrow C$ be a uniformly continuous asymptotically $\kappa$-strict pseudocontractive mapping in the intermediate sense for some $0 \leq \kappa<1$ with sequences $\left\{\gamma_{n}\right\} \subset[0, \infty)$ and $\left\{c_{n}\right\} \subset[0, \infty)$. Let $\left\{T_{n}\right\}_{n=1}^{\infty}$ be a sequence of nonexpansive selfmappings on $C$ and $\left\{\lambda_{n}\right\}$ be a sequence in $(0, b]$ for some $b \in(0,1)$. Let $V$ be a $\bar{\gamma}$-strongly positive bounded linear operator and $f: H \rightarrow H$ be an l-Lipschitzian mapping with $\gamma l<$ $(1+\mu) \bar{\gamma}$. Assume that $\Omega:=\left(\bigcap_{n=1}^{\infty} \operatorname{Fix}\left(T_{n}\right)\right) \cap \operatorname{GMEP}(\Theta, \varphi, A) \cap \mathrm{I}\left(B_{2}, R_{2}\right) \cap \mathrm{I}\left(B_{1}, R_{1}\right) \cap \operatorname{Fix}(S)$ is nonempty. Let $W_{n}$ be the $W$-mapping defined by (1.4) and $\left\{\alpha_{n}\right\},\left\{\beta_{n}\right\}$, and $\left\{\delta_{n}\right\}$ be three sequences in $(0,1)$ such that $0<\kappa+\varepsilon \leq \delta_{n} \leq d<1$. Assume that:

(i) $K: H \rightarrow \mathbf{R}$ is strongly convex with constant $\sigma>0$ and its derivative $K^{\prime}$ is Lipschitz-continuous with constant $v>0$ such that the function $x \mapsto\left\langle y-x, K^{\prime}(x)\right\rangle$ is weakly upper semicontinuous for each $y \in H$;

(ii) for each $x \in H$, there exist a bounded subset $D_{x} \subset C$ and $z_{x} \in C$ such that for any $y \notin D_{x}$,

$$
\Theta\left(y, z_{x}\right)+\varphi\left(z_{x}\right)-\varphi(y)+\frac{1}{r}\left\langle K^{\prime}(y)-K^{\prime}(x), z_{x}-y\right\rangle<0 ;
$$

(iii) $\sum_{n=1}^{\infty}\left(\alpha_{n}+\gamma_{n}+c_{n}\right)<\infty$ and $0<\liminf _{n \rightarrow \infty} \beta_{n} \leq \limsup _{n \rightarrow \infty} \beta_{n}<1$;

(iv) $\left\{\lambda_{i, n}\right\} \subset\left[a_{i}, b_{i}\right] \subset\left(0,2 \eta_{i}\right)$ for $i=1,2$, and $\left\{r_{n}\right\} \subset[0,2 \zeta]$ satisfies

$$
0<\liminf _{n \rightarrow \infty} r_{n} \leq \limsup _{n \rightarrow \infty} r_{n}<2 \zeta
$$

Pick any $x_{1} \in H$ and let $\left\{x_{n}\right\}$ be a sequence generated by the following algorithm:

$$
\left\{\begin{array}{l}
u_{n}=S_{r_{n}}^{(\Theta, \varphi)}\left(I-r_{n} A\right) x_{n}, \\
z_{n}=J_{R_{2}, \lambda_{2, n}}\left(I-\lambda_{2, n} B_{2}\right) J_{R_{1}, \lambda_{1, n}}\left(I-\lambda_{1, n} B_{1}\right) u_{n}, \\
k_{n}=\delta_{n} z_{n}+\left(1-\delta_{n}\right) S^{n} z_{n}, \\
x_{n+1}=\alpha_{n}\left(u+\gamma f\left(x_{n}\right)\right)+\beta_{n} k_{n}+\left(\left(1-\beta_{n}\right) I-\alpha_{n}(I+\mu V)\right) W_{n} z_{n}, \quad \forall n \geq 1 .
\end{array}\right.
$$

Then $\left\{x_{n}\right\}$ converges weakly to $w=\lim _{n \rightarrow \infty} P_{\Omega} x_{n}$ provided $S_{r}^{(\Theta, \varphi)}$ is firmly nonexpansive.

Corollary 4.2 Let C be a nonempty closed convex subset of a real Hilbert space $H$. Let $\Theta$ be a bifunction from $C \times C$ to $\mathbf{R}$ satisfying $(\mathrm{H} 1)-(\mathrm{H} 4)$ and $\varphi: C \rightarrow \mathbf{R}$ be a lower semicontinuous and convex functional. Let $R: C \rightarrow 2^{H}$ be a maximal monotone mapping and let $A: H \rightarrow H$ and $B: C \rightarrow H$ be $\zeta$-inverse-strongly monotone and $\xi$-inverse-strongly monotone, respectively. Let $S: C \rightarrow C$ be a uniformly continuous asymptotically $\kappa$-strict pseudocontractive mapping in the intermediate sense for some $0 \leq \kappa<1$ with sequences $\left\{\gamma_{n}\right\} \subset[0, \infty)$ and 
$\left\{c_{n}\right\} \subset[0, \infty)$. Let $\left\{T_{n}\right\}_{n=1}^{\infty}$ be a sequence of nonexpansive self-mappings on $C$ and $\left\{\lambda_{n}\right\}$ be a sequence in $(0, b]$ for some $b \in(0,1)$. Let $V$ be a $\bar{\gamma}$-strongly positive bounded linear operator and $f: H \rightarrow H$ be an l-Lipschitzian mapping with $\gamma l<(1+\mu) \bar{\gamma}$. Assume that $\Omega:=\left(\bigcap_{n=1}^{\infty} \operatorname{Fix}\left(T_{n}\right)\right) \cap \operatorname{GMEP}(\Theta, \varphi, A) \cap \mathrm{I}(B, R) \cap \operatorname{Fix}(S)$ is nonempty. Let $W_{n}$ be the $W$ mapping defined by (1.4) and $\left\{\alpha_{n}\right\},\left\{\beta_{n}\right\}$, and $\left\{\delta_{n}\right\}$ be three sequences in $(0,1)$ such that $0<\kappa+\varepsilon \leq \delta_{n} \leq d<1$. Assume that:

(i) $K: H \rightarrow \mathbf{R}$ is strongly convex with constant $\sigma>0$ and its derivative $K^{\prime}$ is Lipschitz-continuous with constant $v>0$ such that the function $x \mapsto\left\langle y-x, K^{\prime}(x)\right\rangle$ is weakly upper semicontinuous for each $y \in H$;

(ii) for each $x \in H$, there exist a bounded subset $D_{x} \subset C$ and $z_{x} \in C$ such that for any $y \notin D_{x}$,

$$
\Theta\left(y, z_{x}\right)+\varphi\left(z_{x}\right)-\varphi(y)+\frac{1}{r}\left\langle K^{\prime}(y)-K^{\prime}(x), z_{x}-y\right\rangle<0
$$

(iii) $\sum_{n=1}^{\infty}\left(\alpha_{n}+\gamma_{n}+c_{n}\right)<\infty$ and $0<\liminf _{n \rightarrow \infty} \beta_{n} \leq \lim \sup _{n \rightarrow \infty} \beta_{n}<1$;

(iv) $\left\{\rho_{n}\right\} \subset[a, b] \subset(0,2 \xi)$, and $\left\{r_{n}\right\} \subset[0,2 \zeta]$ satisfies

$$
0<\liminf _{n \rightarrow \infty} r_{n} \leq \limsup _{n \rightarrow \infty} r_{n}<2 \zeta
$$

Pick any $x_{1} \in H$ and let $\left\{x_{n}\right\}$ be a sequence generated by the following algorithm:

$$
\left\{\begin{aligned}
u_{n}= & S_{r_{n}}^{(\Theta, \varphi)}\left(I-r_{n} A\right) x_{n}, \\
k_{n}= & \delta_{n} J_{R, \rho_{n}}\left(I-\rho_{n} B\right) u_{n}+\left(1-\delta_{n}\right) S^{n} J_{R, \rho_{n}}\left(I-\rho_{n} B\right) u_{n}, \\
x_{n+1}= & \alpha_{n}\left(u+\gamma f\left(x_{n}\right)\right)+\beta_{n} k_{n}+\left(\left(1-\beta_{n}\right) I\right. \\
& \left.-\alpha_{n}(I+\mu V)\right) W_{n} J_{R, \rho_{n}}\left(I-\rho_{n} B\right) u_{n}, \quad \forall n \geq 1 .
\end{aligned}\right.
$$

Then $\left\{x_{n}\right\}$ converges weakly to $w=\lim _{n \rightarrow \infty} P_{\Omega} x_{n}$ provided $S_{r}^{(\Theta, \varphi)}$ is firmly nonexpansive.

\section{Competing interests}

The authors declare that they have no competing interests.

\section{Authors' contributions}

All authors contributed equally and significantly in writing this article. All authors read and approved the final manuscript.

\section{Author details}

${ }^{1}$ Department of Mathematics, Shanghai Normal University, Shanghai, 200234, P.R. China. ${ }^{2}$ Scientific Computing Key Laboratory of Shanghai Universities, Shanghai, 200234, P.R. China. ${ }^{3}$ Department of Applied Mathematics, National Hsinchu University of Education, Hsinchu, 30033, Taiwan. ${ }^{4}$ Department of Information Management, Yuan Ze University, Chung-Li, 32003, Taiwan. ${ }^{5}$ Innovation Center for Big Data and Digital Convergence, Yuan Ze University, Chung-Li, 32003, Taiwan.

\section{Acknowledgements}

In this research, first author was partially supported by the National Science Foundation of China (11071169), Innovation Program of Shanghai Municipal Education Commission (09ZZ133) and Ph.D. Program Foundation of Ministry of Education of China (20123127110002). The second author and third author were supported partly by the National Science Council of the Republic of China.

Received: 14 August 2014 Accepted: 14 October 2014 Published: 21 Nov 2014

\section{References}

1. Peng, JW, Yao, JC: A new hybrid-extragradient method for generalized mixed equilibrium problems, fixed point problems and variational inequality problems. Taiwan. J. Math. 12, 1401-1432 (2008) 
2. Ceng, LC, Hu, HY, Wong, MM: Strong and weak convergence theorems for generalized mixed equilibrium problem with perturbation and fixed point problem of infinitely many nonexpansive mappings. Taiwan. J. Math. 15(3), 1341-1367 (2011)

3. Yao, Y, Liou, YC, Yao, JC: New relaxed hybrid-extragradient method for fixed point problems, a general system of variational inequality problems and generalized mixed equilibrium problems. Optimization 60(3), 395-412 (2011)

4. $\mathrm{Cai}, \mathrm{G}, \mathrm{Bu}, \mathrm{SQ}$ : Strong and weak convergence theorems for general mixed equilibrium problems and variational inequality problems and fixed point problems in Hilbert spaces. J. Comput. Appl. Math. 247, 34-52 (2013)

5. Yao, Y, Cho, YJ, Liou, YC: Algorithms of common solutions for variational inclusions, mixed equilibrium problems and fixed point problems. Eur. J. Oper. Res. 212, 242-250 (2011)

6. Ceng, LC, Ansari, QH, Schaible, S: Hybrid extragradient-like methods for generalized mixed equilibrium problems, system of generalized equilibrium problems and optimization problems. J. Glob. Optim. 53, 69-96 (2012)

7. Ceng, LC, Guu, SM, Yao, JC: Hybrid iterative method for finding common solutions of generalized mixed equilibrium and fixed point problems. Fixed Point Theory Appl. 2012, Article ID 92 (2012)

8. Ceng, LC, Petrusel, A: Relaxed extragradient-like method for general system of generalized mixed equilibria and fixed point problem. Taiwan. J. Math. 16(2), 445-478 (2012)

9. Rockafellar, RT: Monotone operators and the proximal point algorithms. SIAM J. Control Optim. 14, 877-898 (1976)

10. Huang, NJ: A new completely general class of variational inclusions with noncompact valued mappings. Comput. Math. Appl. 35(10), 9-14 (1998)

11. Zeng, LC, Guu, SM, Yao, JC: Characterization of H-monotone operators with applications to variational inclusions. Comput. Math. Appl. 50(3-4), 329-337 (2005)

12. Ceng, LC, Guu, SM, Yao, JC: Iterative approximation of solutions for a class of completely generalized set-valued quasi-variational inclusions. Comput. Math. Appl. 56, 978-987 (2008)

13. Ceng, LC, Guu, SM, Yao, JC: Iterative algorithm for finding approximate solutions of mixed quasi-variational-like inclusions. Comput. Math. Appl. 56, 942-952 (2008)

14. Ceng, LC, Ansari, QH, Wong, MM, Yao, JC: Mann type hybrid extragradient method for variational inequalities, variational inclusions and fixed point problems. Comput. Math. Appl. 13(2), 403-422 (2012)

15. Ahmad, R, Ansari, QH, Irfan, SS: Generalized variational inclusions and generalized resolvent equations in Banach spaces. Comput. Math. Appl. 29, 1825-1835 (2005)

16. Ahmad, $\mathrm{R}$, Ansari, $\mathrm{QH}$ : Generalized variational inclusions and $H$-resolvent equations with $H$-accretive operators. Taiwan. J. Math. 111, 703-716 (2007)

17. Ahmad, R, Ansari, QH: System of vector quasi-variational inclusions with some applications. Nonlinear Anal. 69 2812-2824 (2008)

18. Kim, TH, Xu, HK: Convergence of the modified Mann's iteration method for asymptotically strict pseudocontractions. Nonlinear Anal. 68, 2828-2836 (2008)

19. Sahu, DR, Xu, HK, Yao, JC: Asymptotically strict pseudocontractive mappings in the intermediate sense. Nonlinear Anal. 70, 3502-3511 (2009)

20. Ceng, LC, Yao, JC: A hybrid iterative scheme for mixed equilibrium problems and fixed point problems. J. Comput. Appl. Math. 214, 186-201 (2008)

21. Ceng, LC, Yao, JC: A relaxed extragradient-like method for a generalized mixed equilibrium problem, a general system of generalized equilibria and a fixed point problem. Nonlinear Anal. 72, 1922-1937 (2010)

22. Osilike, MO, Aniagbosor, SC, Akuchu, BG: Fixed points of asymptotically demicontractive mappings in arbitrary Banach space. Panam. Math. J. 12, 77-88 (2002)

23. Opial, Z: Weak convergence of the sequence of successive approximations for nonexpansive mappings. Bull. Am. Math. Soc. 73, 591-597 (1967)

24. Huang, S: Hybrid extragradient methods for asymptotically strict pseudo-contractions in the intermediate sense and variational inequality problems. Optimization 60, $739-754$ (2011)

25. Martinez-Yanes, C, Xu, HK: Strong convergence of the CQ method for fixed point iteration process. Nonlinear Anal. 64 2400-2411 (2006)

26. O'Hara, JG, Pillay, P, Xu, HK: Iterative approaches to convex feasibility problems in Banach spaces. Nonlinear Anal. 64(9), 2022-2042 (2006)

27. Yao, Y, Liou, YC, Yao, JC: Convergence theorem for equilibrium problems and fixed point problems of infinite family of nonexpansive mappings. Fixed Point Theory Appl. 2007, Article ID 64363 (2007)

28. Goebel, K, Kirk, WA: Topics on Metric Fixed-Point Theory. Cambridge University Press, Cambridge (1990)

29. Yao, Y, Noor, MA, Zainab, S, Liou, YC: Mixed equilibrium problems and optimization problems. J. Math. Anal. Appl. 354 319-329 (2009)

10.1186/1029-242X-2014-462

Cite this article as: Ceng et al.: On a finite family of variational inclusions with the constraints of generalized mixed equilibrium and fixed point problems. Journal of Inequalities and Applications 2014, 2014:462 